NBI-HE-00-21

ITEP-TH- $11 / 00$

hep-th/0004147

April 2000

\title{
Lattice Gauge Fields and Discrete Noncommutative Yang-Mills Theory
}

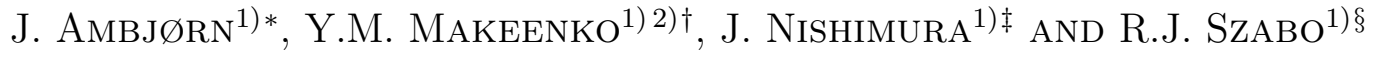 \\ 1) The Niels Bohr Institute \\ Blegdamsvej 17, DK-2100 Copenhagen Ø, Denmark \\ 2) Institute of Theoretical and Experimental Physics \\ B. Cheremushkinskaya 25, 117218 Moscow, Russia
}

\begin{abstract}
We present a lattice formulation of noncommutative Yang-Mills theory in arbitrary even dimensionality. The UV/IR mixing characteristic of noncommutative field theories is demonstrated at a completely nonperturbative level. We prove a discrete Morita equivalence between ordinary Yang-Mills theory with multi-valued gauge fields and noncommutative Yang-Mills theory with periodic gauge fields. Using this equivalence, we show that generic noncommutative gauge theories in the continuum can be regularized nonperturbatively by means of ordinary lattice gauge theory with 't Hooft flux. In the case of irrational noncommutativity parameters, the rank of the gauge group of the commutative lattice theory must be sent to infinity in the continuum limit. As a special case, the construction includes the recent description of noncommutative Yang-Mills theories using twisted large $N$ reduced models. We study the coupling of noncommutative gauge fields to matter fields in the fundamental representation of the gauge group using the lattice formalism. The large mass expansion is used to describe the physical meaning of Wilson loops in noncommutative gauge theories. We also demonstrate Morita equivalence in the presence of fundamental matter fields and use this property to comment on the calculation of the beta-function in noncommutative quantum electrodynamics.
\end{abstract}

\footnotetext{
${ }^{*}$ e-mail address : ambjorn@nbi.dk

$\dagger$ e-mail address : makeenko@nbi.dk

$\ddagger$ Permanent address : Department of Physics, Nagoya University, Nagoya 464-8602, Japan e-mail address : nisimura@nbi.dk

$\S$ e-mail address : szabo@nbi.dk
} 


\section{Contents}

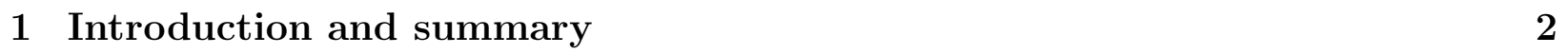

\begin{tabular}{|lll}
2 & Quantum field theory on noncommutative spaces & 6
\end{tabular}

2.1 Scalar field theory $\ldots \ldots \ldots$. . . . . . . . . . . . . . 6

2.2 Noncommutative Yang-Mills theory . . . . . . . . . . . . . . . . . . . . . . 9

2.3 Star-gauge invariant observables . . . . . . . . . . . . . . . . . . . . . . . . . 10

2.4 The noncommutative torus . . . . . . . . . . . . . . . . . . 13

3 Morita equivalence 14

3.1 Twisted gauge theory on the noncommutative torus . . . . . . . . . . . 15

3.2 Transformation of the action . . . . . . . . . . . . . . . . . . . . . 17

$3.3 \quad$ Star-gauge symmetry and transformation of observables . . . . . . . . . . . . 23

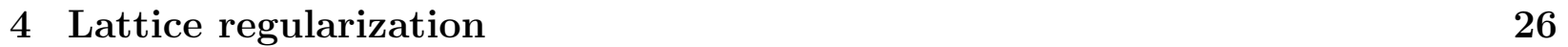

4.1 General construction . . . . . . . . . . . . . . . . . . . . . . 27

4.2 Noncommutative Yang-Mills theory on the lattic . . . . . . . . . . . . . . 31

5 Explicit realizations of discrete noncommutative gauge theory 33

5.1 Discrete Morita equivalence . . . . . . . . . . . . . . . . . . . . . . 34

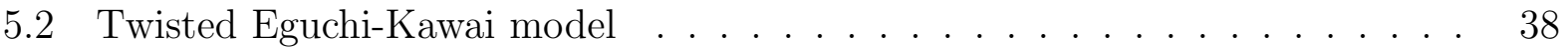

6 Coupling to fundamental matter fields $\quad 40$

6.1 Properties of star-gauge invariant observables . . . . . . . . . . . . . . . . . 41

6.2 Morita equivalence with fundamental matter fields . . . . . . . . . . . . . . . 44

\section{Introduction and summary}

The foundations of noncommutative geometry [1, 2] are inspired in large part by the fundamental principles of quantum mechanics. Just as in quantum mechanics physical observables are replaced with operators, and thereby satisfy the Heisenberg uncertainty principle, in noncommutative geometry spacetime coordinates are replaced with noncommuting operators. The corresponding smearing of spacetime coordinates in this way fits nicely into the ideas behind spacetime uncertainty relations [3] and the concept of minimum length in string theory [4]. This heuristic correspondence has been used to suggest that noncommutative 
geometry provides a natural framework to describe nonperturbative aspects of string theory [2, 5]. This belief is further supported by the fact that Matrix Theory [6] and the IIB matrix model [7], which are conjectured to provide nonperturbative definitions of string theories, give rise to noncommutative Yang-Mills theory on toroidal compactifications [8]. The particular noncommutative toroidal compactification is interpreted as being the result of the presence of a background Neveu-Schwarz two-form field, and it can also be understood in the context of open string quantization in D-brane backgrounds [9, 10]. Furthermore, in Ref. [11] it has been shown that the IIB matrix model with D-brane backgrounds is described by noncommutative Yang-Mills theory.

The early motivation [12] for studying quantum field theory on noncommutative spacetimes was that, because of the spacetime uncertainty relation, the introduction of noncommutativity would provide a natural ultraviolet regularization. However, more recent perturbative calculations [13]-16] have shown that planar noncommutative Feynman diagrams contain exactly the same ultraviolet divergences that their commutative counterparts do, which implies that the noncommutativity does not serve as an ultraviolet regulator. One therefore needs to introduce some other form of regularization to study the dynamics of noncommutative field theories. On the other hand, it has been found that the ultraviolet divergences in non-planar Feynman diagrams [16, 17] exhibit an intriguing mixing of ultraviolet and infrared scales, which can also be described using string-theoretical approaches 18, 19. Heuristically, this UV/IR mixing can be understood in terms of the induced uncertainty relations among the spacetime coordinates. If one measures a given spacetime coordinate with some high precision, then the remaining spacetime directions will generally extend because of the smearing. Furthermore, noncommutative solitons which do not have counterparts in ordinary field theory have been discovered [20] for sufficiently large values of the noncommutativity parameters, and it has also been shown [19] that noncommutative Yang-Mills theory in four dimensions naturally includes gravity.

In order to investigate further the non-trivial dynamics of noncommutative field theories, it is important therefore to develop a nonperturbative regularization of these theories. Such a program has been put forward in Refs. [11, 15, 19, 21]- [24] and it is similar to earlier works 25] based on the mapping between large $N$ matrices and spacetime fields. In particular, in Ref. [22] a unified framework was presented which naturally interpolates between the two ways that noncommutative Yang-Mills theory has appeared in the context of matrix model formulations of string theory, namely the compactification of Matrix theory and the twisted large $N$ reduced model. The model proposed was a finite $N$ matrix model defined by the twisted Eguchi-Kawai model [26, 27] with a quotient condition analogous to the ones considered in Refs. [8, 28]. It was interpreted as a lattice formulation of noncommutative 
gauge theory with manifest star-gauge invariance. The formulation naturally includes Wilson lattice gauge theory [29] for a particular choice of parameters even at finite $N$. In Ref. [24], the lattice formulation was reconsidered from a general point of view without specifying any representation of the noncommutative algebra generated by the spacetime coordinates. This enabled an extension of the formalism to arbitrary even dimensions and also to allow for the minimal coupling to matter fields in the fundamental representation of the gauge group. Furthermore, in this approach one produces finite dimensional representations of the noncommutative geometry in much the same spirit as Weyl's finite version of quantum mechanics [30]. Discrete versions of noncommutative geometry using random lattices and graphs, as well as their applications to finite dimensional versions of noncommutative gauge theories, have also been studied in Ref. [31.

In this paper we will show further that noncommutative Yang-Mills theory in an arbitrary even number of spacetime dimensions, with either rational or irrational valued (dimensionless) noncommutativity parameters and with gauge fields of arbitrary topological charge, can be regularized nonperturbatively by using commutative lattice gauge theories with twisted boundary conditions on the gauge fields [28, 32]. We will do so by presenting an extensive description of the lattice formulation of noncommutative gauge theories which was reported in Ref. 24]. The first striking fact we will find in the general lattice formulation of noncommutative field theories is that the discretization of spacetime inevitably requires that it be compactified, in order to satisfy certain consistency conditions on the noncommutative algebra generated by the spacetime coordinates. It then follows that the lattice spacing must be much smaller than the length scale determined by the noncommutativity parameters, and so the continuum limit does not commute with the commutative limit. This demonstates the $\mathrm{UV} / \mathrm{IR}$ mixing mentioned above at a completely nonperturbative level. We will also show that the dimensionless noncommutativity parameters are necessarily rational-valued on the lattice, which leads immediately to the possibility of obtaining finite dimensional representations of the noncommutative algebra as alluded to above. Irrational noncommutativity parameters can then be obtained by taking the continuum limit appropriately.

Using the lattice formulation, we can regularize noncommutative Yang-Mills theories with periodic boundary conditions on the gauge fields, arbitrary noncommutativity parameters and arbitrary spacetime periodicities. We will then demonstrate on the lattice the Morita equivalence [33] between ordinary, commutative Yang-Mills theory with twisted boundary conditions on the gauge fields and noncommutative Yang-Mills theory with periodic gauge fields. Using this equivalence, we will find that noncommutative Yang-Mills theory with single-valued gauge fields can actually be regularized by means of commutative lattice gauge theory with background magnetic flux. In order to obtain a continuum field theory with 
irrational-valued dimensionless noncommutativity parameters, the rank of the gauge group of the regulating commutative theory must be taken to infinity as one takes the continuum limit. As a special case, the construction includes the recent proposal of using twisted large $N$ reduced models [11] for a concrete definition of noncommutative gauge theory. We shall find, however, that the class of noncommutative Yang-Mills theories that one can obtain using twisted large $N$ reduced models is not the most general one.

The formalism also allows for the coupling of gauge fields to matter fields in the fundamental representation of the gauge group. We will carry out a large mass expansion of the matter-coupled theory and clarify the physical meaning of Wilson loops in noncommutative gauge theories [15, 22, 24, 34, 35]. A remarkable property of these Wilson loops [15] is that star-gauge invariance does not require that the loop be closed, but it does require that the relative distance vector between the two ends of the loop be proportional to its total momentum. Namely, as one increases the total momentum, the loop extends in spacetime. This is another manifestation of UV/IR mixing due to the noncommutativity. We will demonstrate how noncommutative Wilson loops naturally arise from matter field averages in noncommutative gauge theory. By calculating the effective action induced by matter and star-gauge invariant observables constructed out of the matter fields, we shall see that noncommutative Wilson loops play a very fundamental role, just like ordinary Wilson loops do in commutative gauge theories [29]. We will also show explicitly how star-gauge invariant observables reduce smoothly to ordinary gauge invariant observables in the commutative limit for fixed gauge backgrounds.

Morita equivalence in the presence of fundamental matter fields is also proven. As a special case, we obtain the twisted Eguchi-Kawai model with fundamental matter which was introduced in Ref. [36] as a model which reproduces ordinary large $N$ gauge theory in the Veneziano limit. These Morita equivalences clarify the interpretations of various quantities in noncommutative gauge theory. For instance, it is known that only planar noncommutative Feynman diagrams contribute to the beta-function at one-loop order [14, 16, 37], and in the case of noncommutative quantum electrodynamics it is given by [37]

$$
\beta\left(g^{2}\right)=-\frac{g^{4}}{4 \pi^{2}}\left(\frac{11}{3}-\frac{2}{3} n_{f}\right),
$$

where $n_{f}$ is the number of fermion flavours. The beta-function (1.1) coincides with that of ordinary $\mathrm{U}(N)$ Yang-Mills theory coupled to $N_{f}=n_{f} N$ flavours of fermion fields in the large $N$ (planar) limit (after an appropriate rescaling of the Yang-Mills coupling constant $g$ ). This coincidence can be understood as a consequence of the Morita equivalence that we shall derive. In fact, the results of such calculations indicate that the phenomenon of Morita equivalence in noncommutative gauge theories holds beyond the classical level in regularized 
perturbation theory. We will further show that the star-gauge invariant observables of two Morita equivalent noncommutative Yang-Mills theories are in a one-to-one correspondence. Owing to this fact, we can define regularized correlation functions of star-gauge invariant observables in noncommutative Yang-Mills theory with multi-valued gauge fields by using the lattice regularization of the corresponding Morita equivalent noncommutative gauge theory with periodic gauge fields. This Morita equivalence property will be the key feature which permits the discretization of generic noncommutative Yang-Mills theories in the continuum.

The organization of the remainder of this paper is as follows. In Section 2 we present a pedagogical introduction to noncommutative field theory, and in particular noncommutative Yang-Mills theory, for the sake of completeness and in order to set up the notations to be used throughout the paper. In Section 3, we present a detailed and very general field theoretical derivation of the Morita equivalence relation between noncommutative gauge theories and demonstrate the one-to-one correspondence between star-gauge invariant observables in the Morita equivalent theories. In Section 1 , we construct the discrete version of noncommutative field theory. We show that the lattice formulation exhausts the noncommutative Yang-Mills theories in arbitrary even dimensions, given the Morita equivalence properties described in Section 3. In Section 5 we establish Morita equivalence on the lattice, which allows us to regularize noncommutative Yang-Mills theories by means of commutative lattice gauge theories with twisted boundary conditions on the gauge fields. We also describe the relationship to finite dimensional matrix model representations of noncommutative gauge theories. Finally, in Section 6 we introduce matter fields in the fundamental representation

of the gauge group and study the properties of star-gauge invariant observables as well as Morita equivalence in the presence of matter fields.

\section{Quantum field theory on noncommutative spaces}

In this Section we will briefly review some aspects of constructing noncommutative gauge theories in the continuum, in a way that will enable us to later construct a lattice version of the field theory. We will start with a simple scalar field theory to introduce the ideas, and then move on to a description of noncommutative Yang-Mills theory and its observables.

\subsection{Scalar field theory}

Consider the scalar quantum field theory which is described by the partition function

$$
\begin{aligned}
Z & =\int \mathcal{D} \phi \mathrm{e}^{-S[\phi]} \\
S & =\int \mathrm{d}^{D} x\left(\frac{1}{2}\left(\partial_{\mu} \phi\right)^{2}+\frac{1}{2} \phi^{2}+\frac{1}{4 !} \phi^{4}\right),
\end{aligned}
$$


where $\phi(x)$ is a real-valued scalar field on $D$-dimensional Euclidean spacetime $\mathbb{R}^{D}$. For fields in a Schwartz space of functions of sufficiently rapid decrease at infinity, we may use the Fourier transformation

$$
\phi(x)=\int \frac{\mathrm{d}^{D} k}{(2 \pi)^{D}} \tilde{\phi}(k) \mathrm{e}^{i k_{\mu} x_{\mu}},
$$

with $\tilde{\phi}(-k)=\tilde{\phi}(k)^{*}$. The first step in generalizing a quantum field theory on an ordinary spacetime to one on a noncommutative spacetime is to replace the local coordinates $x_{\mu}$ by hermitian operators $\hat{x}_{\mu}$ obeying the commutation relations

$$
\left[\hat{x}_{\mu}, \hat{x}_{\nu}\right]=i \theta_{\mu \nu}
$$

where $\theta_{\mu \nu}=-\theta_{\nu \mu}$ are real-valued c-numbers with dimensions of length squared. Consequently, fields on spacetime are replaced by operators. Replacing $x_{\mu}$ in (2.2) by $\hat{x}_{\mu}$, we obtain

$$
\hat{\phi}=\int \frac{\mathrm{d}^{D} k}{(2 \pi)^{D}} \tilde{\phi}(k) \mathrm{e}^{i k_{\mu} \hat{x}_{\mu}} .
$$

The operator-ordering ambiguity which exists when we replace $x_{\mu}$ by $\hat{x}_{\mu}$ is fixed in (2.4) by requiring covariance and hermiticity of the operator $\hat{\phi}$. The operator (2.4) is called a Weyl operator or the Weyl symbol of the field $\phi(x)$, and this method of constructing a noncommutative field theory is sometimes refered to as Weyl quantization.

Combining (2.2) and (2.4), one can obtain an explicit map $\hat{\Delta}(x)$ which transforms a field $\phi(x)$ to an operator $\hat{\phi}$ as

$$
\begin{aligned}
\hat{\phi} & =\int \mathrm{d}^{D} x \phi(x) \hat{\Delta}(x) \\
\hat{\Delta}(x) & =\int \frac{\mathrm{d}^{D} k}{(2 \pi)^{D}} \mathrm{e}^{i k_{\mu} \hat{x}_{\mu}} \mathrm{e}^{-i k_{\mu} x_{\mu}} .
\end{aligned}
$$

This means that the field $\phi(x)$ can be thought of as the coordinate space representation of the operator $\hat{\phi}$. Note that the operator (2.6) is hermitian, $\hat{\Delta}(x)^{\dagger}=\hat{\Delta}(x)$, and in the commutative case $\theta_{\mu \nu}=0$ it reduces to a delta-function $\delta^{D}(\hat{x}-x)$. However, for $\theta_{\mu \nu} \neq 0$, $\hat{\Delta}(x)$ is a complicated map. We may also introduce an anti-hermitian derivation $\hat{\partial}_{\mu}$ through the commutation relations

$$
\left[\hat{\partial}_{\mu}, \hat{x}_{\nu}\right]=\delta_{\mu \nu} \quad, \quad\left[\hat{\partial}_{\mu}, \hat{\partial}_{\nu}\right]=i c_{\mu \nu}
$$

where $c_{\mu \nu}$ are real-valued c-numbers.1 One can show that

$$
\begin{aligned}
{\left[\hat{\partial}_{\mu}, \hat{\phi}\right] } & =\int \mathrm{d}^{D} x \partial_{\mu} \phi(x) \hat{\Delta}(x) \\
{\left[\hat{\partial}_{\mu}, \hat{\Delta}(x)\right] } & =-\partial_{\mu} \hat{\Delta}(x) .
\end{aligned}
$$

\footnotetext{
${ }^{1}$ The c-numbers $c_{\mu \nu}$ turn out to be irrelevant for our purposes, since the operator $\hat{\partial}_{\mu}$ will only appear in commutator brackets. For this reason it is conventional to set $c_{\mu \nu}=0$. However, this restriction is not necessary, and indeed we will encounter cases with non-vanishing $c_{\mu \nu}$ later on.
} 
From (2.8) it follows that any translation generator can be represented by the operator $\mathrm{e}^{v_{\mu} \hat{\partial}_{\mu}}, v_{\mu} \in \mathbb{R}$, which satisfies

$$
\mathrm{e}^{v_{\mu} \hat{\partial}_{\mu}} \hat{\Delta}(x) \mathrm{e}^{-v_{\mu} \hat{\partial}_{\mu}}=\hat{\Delta}(x+v) .
$$

The existence of such an operator implies that $\operatorname{Tr} \hat{\Delta}(x)$ is independent of $x$ for any trace Tr on the algebra of operators. Therefore, one can represent an integration of fields on the spacetime as

$$
\operatorname{Tr} \hat{\phi}=\int \mathrm{d}^{D} x \phi(x)
$$

where the normalization of the operator trace is fixed by requiring $\operatorname{Tr} \hat{\Delta}(x)=1$. Using (2.5), (2.6) and (2.10) it is straightforward to show that the collection of operators $\hat{\Delta}(x)$ for $x \in \mathbb{R}^{D}$ form an orthonormal set,

$$
\operatorname{Tr}(\hat{\Delta}(x) \hat{\Delta}(y))=\delta^{D}(x-y),
$$

from which it follows that the inverse of the map (2.6) is given by

$$
\phi(x)=\operatorname{Tr}(\hat{\phi} \hat{\Delta}(x)) .
$$

Therefore, there is a one-to-one correspondence between fields (of sufficiently rapid decrease at infinity) and operators. This can be thought of as an analog of the operator-state correspondence of local quantum field theory.

Using these definitions, one can define a scalar quantum field theory on a noncommutative spacetime as

$$
\begin{aligned}
Z & =\int \mathrm{d} \hat{\phi} \mathrm{e}^{-S[\hat{\phi}]} \\
S[\hat{\phi}] & =\operatorname{Tr}\left(\frac{1}{2}\left[\hat{\partial}_{\mu}, \hat{\phi}\right]^{2}+\frac{1}{2} \hat{\phi}^{2}+\frac{1}{4 !} \hat{\phi}^{4}\right),
\end{aligned}
$$

where the measure $\mathrm{d} \hat{\phi}$ is nothing but the ordinary path integral measure $\mathcal{D} \phi$. The difference between noncommutative quantum field theory and ordinary field theory comes from the different products of fields that are used in the two cases. Suppose that $\hat{\phi}_{3}=\hat{\phi}_{1} \hat{\phi}_{2}$, where $\hat{\phi}_{i}=\int \mathrm{d}^{D} x \phi_{i}(x) \hat{\Delta}(x)$. It is straightforward to compute that the corresponding scalar field $\phi_{3}(x)$ is given by

$$
\begin{aligned}
\phi_{3}(x) & =\operatorname{Tr}\left(\hat{\phi}_{1} \hat{\phi}_{2} \hat{\Delta}(x)\right) \\
& =\frac{1}{\pi^{D}|\operatorname{det} \theta|} \iint \mathrm{d}^{D} y \mathrm{~d}^{D} z \phi_{1}(y) \phi_{2}(z) \mathrm{e}^{-2 i\left(\theta^{-1}\right)_{\mu \nu}(x-y)_{\mu}(x-z)_{\nu}} \\
& =\phi_{1}(x) \exp \left(\frac{i}{2} \overleftarrow{\partial_{\mu}} \theta_{\mu \nu} \overrightarrow{\partial_{\nu}}\right) \phi_{2}(x) \\
& \stackrel{\text { def }}{=} \phi_{1}(x) \star \phi_{2}(x)
\end{aligned}
$$


where in the second line we have assumed that $\theta_{\mu \nu}$ is an invertible matrix. The multiplication in (2.14) is called the star or Moyal product of the fields $\phi_{1}(x)$ and $\phi_{2}(x)$. It is associative but not commutative. For $\theta_{\mu \nu}=0$, it reduces to the ordinary product of functions and the theory (2.13) to the usual scalar field theory (2.1).

One can easily show that $\int \mathrm{d}^{D} x \phi_{i}(x) \star \phi_{j}(x) \star \cdots \star \phi_{k}(x)$ is invariant under cyclic permutation of the fields, justifying the representation of the trace in (2.10). Since

$$
\int \mathrm{d}^{D} x \phi_{1}(x) \star \phi_{2}(x)=\int \mathrm{d}^{D} x \phi_{1}(x) \phi_{2}(x),
$$

noncommutative field theory and ordinary field theory are identical at the level of free fields. The differences come when one considers the interaction term

$$
\begin{aligned}
\operatorname{Tr}\left(\hat{\phi}^{4}\right) & =\int \mathrm{d}^{D} x \phi(x) \star \phi(x) \star \phi(x) \star \phi(x) \\
& =\int\left(\prod_{i=1}^{4} \frac{\mathrm{d}^{D} k_{i}}{(2 \pi)^{D}}\right)(2 \pi)^{D} \delta^{D}\left(\sum_{i=1}^{4} k_{i}\right)\left(\prod_{i=1}^{4} \tilde{\phi}\left(k_{i}\right)\right) \mathrm{e}^{-\frac{i}{2} \theta_{\mu \nu} \sum_{i<j} k_{\mu i} k_{\nu j}} .
\end{aligned}
$$

We see that the vertex in the noncommutative field theory contains a momentum dependent phase factor. The interaction is therefore non-local. Naively one might expect that the effect of the noncommutativity becomes negligible at energy scales much smaller than $\left|\theta_{\mu \nu}\right|^{-1 / 2}$. This is not quite the case. For example, it has been shown that the noncommutativity drastically alters the infrared dynamics of the theory [16, 17]. The perturbative renormalizability of noncommutative scalar field theory has been studied in Ref. [38.

\subsection{Noncommutative Yang-Mills theory}

Let us now turn to gauge theory on a noncommutative spacetime. A hermitian operator corresponding to a $\mathrm{U}(p)$ gauge field $A_{\mu}(x)$ can be introduced as

$$
\hat{A}_{\mu}=\int \mathrm{d}^{D} x \hat{\Delta}(x) \otimes A_{\mu}(x)
$$

where $\hat{\Delta}(x)$ is given by $(2.6)$. The action can be written as

$$
\begin{aligned}
S & =\operatorname{Tr} \operatorname{tr}{ }_{(p)}\left(\left[\hat{\partial}_{\mu}, \hat{A}_{\nu}\right]-\left[\hat{\partial}_{\nu}, \hat{A}_{\mu}\right]+i\left[\hat{A}_{\mu}, \hat{A}_{\nu}\right]\right)^{2} \\
& =\int \mathrm{d}^{D} x \operatorname{tr}{ }_{(p)}\left(F_{\mu \nu}(x) \star F_{\mu \nu}(x)\right)
\end{aligned}
$$

where

$$
F_{\mu \nu}=\partial_{\mu} A_{\nu}-\partial_{\nu} A_{\mu}+i\left(A_{\mu} \star A_{\nu}-A_{\nu} \star A_{\mu}\right)
$$


As in (2.10), we use the symbol $\operatorname{Tr}$ to denote the operator trace over coordinates, while $\operatorname{tr}_{(p)}$ denotes the (finite-dimensional) trace in the fundamental representation of the $\mathrm{U}(p)$ gauge group. The action (2.19) is invariant under the "star-gauge" transformation

$$
A_{\mu}(x) \mapsto g(x) \star A_{\mu}(x) \star g(x)^{\dagger}-i g(x) \star \partial_{\mu} g(x)^{\dagger},
$$

where the gauge function $g(x)$ is a $p \times p$ matrix field satisfying

$$
g(x) \star g(x)^{\dagger}=\mathbb{1}_{p},
$$

namely it is star-unitary. Introducing a unitary operator corresponding to the gauge function $g(x)$ through

$$
\hat{g}=\int \mathrm{d}^{D} x \hat{\Delta}(x) \otimes g(x),
$$

the gauge transformation (2.21) can be written in terms of Weyl operators as

$$
\hat{A}_{\mu} \mapsto \hat{g} \hat{A}_{\mu} \hat{g}^{\dagger}-i \hat{g}\left[\hat{\partial}_{\mu}, \hat{g}^{\dagger}\right],
$$

under which the action (2.18) is invariant.

As can be seen from the action (2.19), three-point and four-point gauge interactions exist even for the simplest case of a $\mathrm{U}(1)$ gauge group. It is known that noncommutative $\mathrm{U}(1)$ gauge theory in four dimensions is asymptotically free, and in fact that its beta-function coincides exactly with that of large $N$ Yang-Mills theory (See eq. (1.1)). This can be understood as a consequence of Morita equivalence of noncommutative gauge theories, which we will derive in Section 2 . One form of Morita equivalence states that the non-abelian structure of the gauge group can be absorbed into the noncommutativity of spacetime. Note in this regard that Eq. (2.17) already has a suggestive form, as it shows that the spacetime indices and the gauge group indices are treated on the same footing in noncommutative geometry.

\subsection{Star-gauge invariant observables}

In Ref. [15], star-gauge invariant observables in noncommutative Yang-Mills theory have been discovered using a twisted large $N$ reduced model. In this subsection, we construct star-gauge invariant observables in the present general formalism following Ref. 222]. For this, we first note that a novel feature of the star-product is that spacetime translations can be represented on fields via star-conjugation by functions on spacetime. Let us consider a star-unitary function $S_{v}(x)$ with the propertyf

$$
S_{v}(x) \star g(x) \star S_{v}(x)^{\dagger}=g(x+v)
$$

\footnotetext{
${ }^{2}$ In principle, $S_{v}(x)$ can be a $p \times p$ matrix-valued function, but we shall find below that it is proportional to the unit matrix.
} 
for arbitrary functions $g(x)$, where $v$ is a real $D$-dimensional vector. It is straightforward to show that a necessary and sufficient condition for such a function to exist is

$$
\theta_{\mu \nu} \partial_{\nu} S_{v}(x)=i v_{\mu} S_{v}(x)
$$

In particular, when $\theta_{\mu \nu}$ is invertible, the solution to (2.26) exists for arbitrary $v_{\mu}$ and is given by the plane wave

$$
S_{v}(x)=\mathrm{e}^{i k_{\mu} x_{\mu}} \mathbb{1}_{p} \quad \text { with } k_{\mu}=\left(\theta^{-1}\right)_{\mu \nu} v_{\nu} .
$$

The uniqueness of the function (2.27) is immediate. If $S_{v}^{\prime}(x)$ is another function with the property (2.25), then the function $S_{v}^{\prime \dagger}(x) \star S_{v}(x)$ star-commutes with all functions $g(x)$. In particular, by taking $g(x)=\mathrm{e}^{i w_{\mu} x_{\mu}}$ for arbitrary $w_{\mu} \in \mathbb{R}$ we may conclude that the function $S_{v}^{\prime \dagger}(x) \star S_{v}(x)$ is independent of $x$, and thus the two functions $S_{v}(x)$ and $S_{v}^{\prime}(x)$ coincide up to some irrelevant phase factor.

We now construct an analog of the parallel transport operator as

$$
\begin{aligned}
\mathcal{U}(x ; C)= & \mathrm{P} \exp _{\star}\left(i \int_{C} \mathrm{~d} \xi^{\mu} A_{\mu}(x+\xi)\right) \\
\stackrel{\text { def }}{=} & 1+\sum_{n=1}^{\infty} i^{n} \int_{0}^{1} \mathrm{~d} \sigma_{1} \int_{\sigma_{1}}^{1} \mathrm{~d} \sigma_{2} \cdots \int_{\sigma_{n-1}}^{1} \mathrm{~d} \sigma_{n} \xi^{\prime} \mu_{1}\left(\sigma_{1}\right) \cdots \xi^{\prime} \mu_{n}\left(\sigma_{n}\right) \\
& \times A_{\mu_{1}}\left(x+\xi\left(\sigma_{1}\right)\right) \star \cdots \star A_{\mu_{n}}\left(x+\xi\left(\sigma_{n}\right)\right)
\end{aligned}
$$

where $C$ is an oriented curve in $D$-dimensional spacetime parametrized by the functions $\xi_{\mu}(\sigma)$ with $0 \leq \sigma \leq 1$. We fix the starting point of the curve to be the origin, $\xi_{\mu}(0)=0$, and denote its endpoint by $v_{\mu}=\xi_{\mu}(1)$ in what follows. The operator $\mathcal{U}(x ; C)$ can be regarded as a $p \times p$ star-unitary matrix field depending on $C$. Under the star-gauge transformation (2.21), it transforms as

$$
\mathcal{U}(x ; C) \mapsto g(x) \star \mathcal{U}(x ; C) \star g(x+v)^{\dagger}
$$

Star-gauge invariant observables can be constructed out of (2.28) by using the function (2.27), with the property (2.25), as

$$
\mathcal{O}(C)=\int \mathrm{d}^{D} x \operatorname{tr}_{(p)}\left(\mathcal{U}(x ; C) \star S_{v}(x)\right) .
$$

In Eq. (2.30), the parameter $k_{\mu}$ in (2.27) can be interpreted as the total momentum of the contour $C$. Note that in the commutative case $\theta_{\mu \nu}=0$, gauge invariance requires that the loop be closed irrespectively of the total momentum. In the noncommutative case, on the other hand, star-gauge invariance does not require that the loop be closed, but it does require 
the separation vector $v_{\mu}$ of the loop to be proportional to the total momentum, as is seen from (2.27). The larger the total momentum is, the longer becomes the open loop. We will study further the dynamics of noncommutative Wilson loops in Section 6.1 by introducing matter fields as probes.

Let us now rewrite the star-gauge invariant quantity (2.30) in terms of Weyl operators. After a little algebra, the parallel transport operator can be rewritten as

$$
\mathcal{U}(x ; C)=\operatorname{Tr}\left(\hat{U}(C) \hat{D}(C)^{\dagger} \hat{\Delta}(x)\right)
$$

where we have introduced the unitary operators

$$
\begin{aligned}
& \hat{U}(C)=\mathrm{P} \exp \left(\int_{C} \mathrm{~d} \xi^{\mu}\left(\hat{\partial}_{\mu}+i \hat{A}_{\mu}\right)\right), \\
& \hat{D}(C)=\mathrm{P} \exp \left(\int_{C} \mathrm{~d} \xi^{\mu} \hat{\partial}_{\mu}\right) .
\end{aligned}
$$

Under a gauge transformation $(2.24), \hat{U}(C)$ transforms as

$$
\hat{U}(C) \mapsto \hat{g} \hat{U}(C) \hat{g}^{\dagger}
$$

Note that $\hat{D}(C)$ is nothing but a translation operator (up to an irrelevant phase factor),

$$
\hat{D}(C) \hat{\Delta}(x) \hat{D}(C)^{\dagger}=\hat{\Delta}(x+v)
$$

We also introduce the unitary operator

$$
\hat{S}_{v}=\int \mathrm{d}^{D} x \hat{\Delta}(x) \otimes S_{v}(x)=\mathrm{e}^{i k_{\mu} \hat{x}_{\mu}} \otimes \mathbb{1}_{p}
$$

which satisfies

$$
\hat{S}_{v} \hat{\Delta}(x) \hat{S}_{v}^{\dagger}=\hat{\Delta}(x+v) \otimes \mathbb{1}_{p}
$$

corresponding to (2.25). The Weyl operator description of star-gauge invariant observables is then given by

$$
\mathcal{O}(C)=\operatorname{Tr} \operatorname{tr}_{(p)}\left(\hat{U}(C) \hat{D}(C)^{\dagger} \hat{S}_{v}\right)
$$

Its gauge invariance can be checked directly by noting that the operator $\hat{D}(C)^{\dagger} \hat{S}_{v}$ in 2.37 ) commutes with $\hat{\Delta}(x)$ due to (2.34) and (2.36). 


\subsection{The noncommutative torus}

We now briefly discuss the case where the spacetime is a $D$-dimensional torus $\mathbb{T}^{D}$ instead of $\mathbb{R}^{D}$. Let us first consider the case discussed in subsection 2.1 and impose periodic boundary conditions on the scalar field $\phi(x)$ (Twisted boundary conditions will be studied in the next Section),

$$
\phi\left(x+\Sigma_{\mu a} \hat{\mu}\right)=\phi(x), \quad a=1, \ldots, D,
$$

where $\hat{\mu}$ is a unit vector in the $\mu$-th direction of spacetime, and $\Sigma_{\mu a}$ is the $D \times D$ period matrix of the torus which is a vielbein for the metric of $\mathbb{T}^{D}$. Here and in the following we use Greek letters for spacetime indices and Latin letters for frame indices. Due to the periodicity (2.38), the momenta $k_{\mu}$ in the Fourier mode expansion (2.2) are quantized as

$$
k_{\mu}=2 \pi\left(\Sigma^{-1}\right)_{a \mu} m_{a}, \quad m_{a} \in \mathbb{Z} .
$$

We define a mapping of fields into operators as in (2.5) but now with the $\hat{\Delta}(x)$ given by

$$
\hat{\Delta}(x)=\frac{1}{|\operatorname{det} \Sigma|} \sum_{\vec{m} \in \mathbb{Z}^{D}}\left(\prod_{a=1}^{D}\left(\hat{Z}_{a}\right)^{m_{a}}\right) \mathrm{e}^{-\pi i \sum_{a<b} \Theta_{a b} m_{a} m_{b}} \mathrm{e}^{-2 \pi i\left(\Sigma^{-1}\right)_{a \mu} m_{a} x_{\mu}}
$$

where the operators

$$
\hat{Z}_{a}=\mathrm{e}^{2 \pi i\left(\Sigma^{-1}\right)_{a \mu} \hat{x}_{\mu}}
$$

satisfy the commutation relations

$$
\begin{aligned}
\hat{Z}_{a} \hat{Z}_{b} & =\mathrm{e}^{-2 \pi i \Theta_{a b}} \hat{Z}_{b} \hat{Z}_{a} \\
{\left[\hat{\partial}_{\mu}, \hat{Z}_{a}\right] } & =2 \pi i\left(\Sigma^{-1}\right)_{a \mu} \hat{Z}_{a}
\end{aligned}
$$

with

$$
\Theta_{a b}=2 \pi\left(\Sigma^{-1}\right)_{a \mu} \theta_{\mu \nu}\left(\Sigma^{-1}\right)_{b \nu}
$$

the dimensionless noncommutativity parameter. The basis (2.40) has the requisite properties

$$
\begin{aligned}
\hat{\Delta}\left(x+\Sigma_{\mu a} \hat{\mu}\right) & =\hat{\Delta}(x), \quad a=1, \ldots, D, \\
{\left[\hat{\partial}_{\mu}, \hat{\Delta}(x)\right] } & =-\partial_{\mu} \hat{\Delta}(x) .
\end{aligned}
$$

Note that the torus $\mathbb{T}^{D}$ has a discrete geometrical symmetry given by its $\operatorname{SL}(D, \mathbb{Z})$ automorphism group, under which the period matrix transforms as $\Sigma \mapsto \Sigma \Lambda$ with $\Lambda \in \operatorname{SL}(D, \mathbb{Z})$. The dimensionless noncommutativity parameter (2.43) transforms as $\Theta \mapsto \Lambda^{-1} \Theta\left(\Lambda^{-1}\right)^{\top}$ and the

coordinate operators (2.41) as $\hat{Z}_{a} \mapsto \prod_{b=1}^{D}\left(\hat{Z}_{b}\right)^{\left(\Lambda^{-1}\right)_{a b}}$. All formulae such as (2.40)-(2.43) are manifestly $\mathrm{SL}(D, \mathbb{Z})$ covariant. This symmetry, which persists in the case of generic twisted boundary conditions on the fields, will be exploited in the next Section. 
Let us now consider star-gauge invariant observables in noncommutative Yang-Mills theory on the torus $\mathbb{T}^{D}[22$. We can define the parallel transport operator $\mathcal{U}(x ; C)$ as in $(2.28)$. The star-gauge transformation of $\mathcal{U}(x ; C)$ is given by (2.29), but now the star-unitary function $g(x)$ is a single-valued function on the torus. We can define a star-gauge invariant observable as in (2.30), where $S_{v}(x)$ is a function on the $D$-dimensional torus which satisfies (2.25) for arbitrary $g(x)$ that we are considering. One finds that a necessary and sufficient condition is

$$
S_{v}\left(x+2 \pi\left(\Sigma^{-1} \theta\right)_{a \mu} \hat{\mu}\right)=\mathrm{e}^{2 \pi i\left(\Sigma^{-1}\right)_{a \mu} v_{\mu}} S_{v}(x) .
$$

The solution can again be given by the plane wave $S_{v}(x)=\mathrm{e}^{i k_{\mu} x_{\mu}} \mathbb{1}_{p}$, but in the present case $k_{\mu}$ is quantized as in (2.39). The condition that must be met is

$$
v_{\mu}=\theta_{\mu \nu} k_{\nu}+\Sigma_{\mu a} n_{a}
$$

for some integer-valued vector $n_{a}$. Thus we obtain an analog of the Polyakov line in noncom-

mutative Yang-Mills theory represented by the existence of the case with non-zero "winding number" $n_{a}$.

\section{Morita equivalence}

In noncommutative geometry, there is a remarkable geometric equivalence relation on certain classes of noncommutative spaces known as Morita equivalence [1]. Roughly speaking, two spaces are Morita equivalent if one space can be regarded as a twisted operator bundle over the other space of a certain topological charge whose fibers are operator algebras. Many topological quantities are preserved by the Morita equivalence relation. In particular, K-theory groups are invariant under it, so that two Morita equivalent spaces should have a canonical mapping between gauge bundles defined over them. In noncommutative Yang-Mills theory, this implies a remarkable duality between gauge theories over different noncommutative tori [33], for example, which relates a Yang-Mills theory with background magnetic flux to a gauge theory with gauge group of lower rank and no background flux. It allows one to interpolate continuously, through noncommutative Yang-Mills theories, between two ordinary Yang-Mills theories with gauge groups of different rank and appropriate background magnetic fluxes. Furthermore, in certain instances, there is the remarkable fact that the non-abelian nature of a gauge group can be absorbed into the noncommutativity of spacetime by mapping a $\mathrm{U}(p)$ gauge theory with multi-valued gauge fields to a $\mathrm{U}(1)$ gauge theory with single-valued fields on a dual noncommutative torus. In string theory, Morita equivalence coincides with the phenomenon of T-duality of open string backgrounds in the presence of D-branes 10, 33. 
The nonperturbative formulation of generic noncommutative Yang-Mills theories that we shall present in Section 4 relies crucially on the Morita equivalence property. We shall therefore present a detailed and rather general derivation of the Morita equivalence relation for noncommutative tori from the point of view adopted in the previous Section to noncommutative Yang-Mills theory. We shall see that, in the present formalism, the equivalence has a natural geometrical interpretation as a change of basis for the map between fields and Weyl operators for the noncommutative geometry. We will also show that this duality holds at the level of star-gauge invariant observables in the two gauge theories, thereby providing a complete equivalence of the noncommutative quantum field theories.

\subsection{Twisted gauge theory on the noncommutative torus}

Consider a $\mathrm{U}(p)$ gauge theory on a noncommutative torus $\mathbb{T}_{\Theta}^{D}$ with gauge fields of nonvanishing topological charge. Such gauge fields are not single-valued functions on the torus. Instead, they must be regarded as functions on the universal covering space $\mathbb{R}^{D}$ with the twisted boundary conditions

$$
A_{\mu}\left(x+\Sigma_{\nu a} \hat{\nu}\right)=\Omega_{a}(x) \star A_{\mu}(x) \star \Omega_{a}(x)^{\dagger}-i \Omega_{a}(x) \star \partial_{\mu} \Omega_{a}(x)^{\dagger}
$$

The transition functions $\Omega_{a}(x)$ are star-unitary $p \times p$ matrices, which we decompose into an abelian part and an $\mathrm{SU}(p)$ part via the gauge choice

$$
\Omega_{a}(x)=\mathrm{e}^{i \alpha_{a \mu} x_{\mu}} \otimes \Gamma_{a}
$$

where $\alpha$ is a real-valued constant $D \times D$ matrix satisfying $(\alpha \Sigma)^{\top}=-\alpha \Sigma$, and $\Gamma_{a}$ are $\mathrm{SU}(p)$ matrices. The matrix $\alpha$ will account for the abelian fluxes of the gauge fields. The antisymmetry of the matrix $\alpha \Sigma$ implies that the transition function $\Omega_{a}(x)$ has periodicity $\Omega_{a}\left(x+\Sigma_{\mu a} \hat{\mu}\right)=\Omega_{a}(x)$.

Consistency of the conditions (3.1) requires the $\Omega_{a}$ to obey the cocycle condition

$$
\Omega_{a}\left(x+\Sigma_{\mu b} \hat{\mu}\right) \star \Omega_{b}(x)=\mathcal{Z}_{a b} \Omega_{b}\left(x+\Sigma_{\mu a} \hat{\mu}\right) \star \Omega_{a}(x),
$$

where $\mathcal{Z}_{a b}=\mathrm{e}^{2 \pi i \gamma_{a b} / p}$ are elements of the center of the $\mathrm{SU}(p)$ part of the gauge group, with $\gamma$ an antisymmetric integral $D \times D$ matrix. In commutative $\mathrm{SU}(p)$ gauge theory, a phase factor such as $\mathcal{Z}_{a b}$ would induce a non-abelian 't Hooft flux for the gauge fields [32]. In that case, the matrix $\alpha$ in (3.2) should be set to zero, and therefore having a non-trivial $\mathcal{Z}_{a b}$ is the only way to twist the boundary conditions on the gauge fields. In commutative $\mathrm{U}(p)$ gauge theory, keeping both $\gamma$ and $\alpha$ non-zero is redundant because one can eliminate the $\mathrm{U}(1)$ twists $\alpha$ by $\mathbb{Z}_{p}$-valued phase factors according to the global decomposition $\mathrm{U}(p)=\mathrm{U}(1) \times \mathrm{SU}(p) / \mathbb{Z}_{p}$, 
and one can set either $\gamma=0$ or $\alpha=0$ without loss of generality. However, this is not quite the case in noncommutative $\mathrm{U}(p)$ gauge theory, as we will see.

Eq. (3.3) implies that the matrices $\Gamma_{a}$ mutually commute up to some phases,

$$
\Gamma_{a} \Gamma_{b}=\mathrm{e}^{2 \pi i Q_{a b} / p} \Gamma_{b} \Gamma_{a}
$$

Taking the determinant of both sides of (3.4) shows that the antisymmetric matrix $Q$ has elements $Q_{a b} \in \mathbb{Z}$. Furthermore, eq. (3.3) gives the consistency condition

$$
Q=\frac{p}{2 \pi}\left(2 \alpha \Sigma-\alpha \theta \alpha^{\top}\right)-\gamma
$$

where here and in the following we use a matrix multiplication convention. The integer $Q_{a b}$ is the magnetic flux of the gauge field through the surface formed by the $a$-th and $b$-th cycles of the torus. The central extension of the cocycle relation (3.3) shifts $Q$ by the matrix $\gamma$ of non-abelian 't Hooft fluxes representing the associated principal curvatures.

The fact that the trivial configuration $A_{\mu}(x)=0$ is not a solution of (3.1) motivates the introduction of a fixed, multi-valued background abelian gauge field $A_{\mu}^{(0)}(x)$ defined by

$$
A_{\mu}^{(0)}(x)=\frac{1}{2} F_{\mu \nu} x_{\nu} \otimes \mathbb{1}_{p}
$$

where $F$ is a real-valued constant antisymmetric $D \times D$ matrix. The twisted boundary conditions (3.1) for the gauge field $A_{\mu}^{(0)}(x)$ are equivalent to the relations

$$
\begin{aligned}
\alpha & =-\Sigma^{\top} F \frac{1}{2 \mathbb{1}_{D}+\theta F} \\
F & =2 \alpha^{\top} \frac{1}{\Sigma-\theta \alpha^{\top}} .
\end{aligned}
$$

We then decompose $A_{\mu}(x)$ into a part representing a particular solution to the twisted boundary conditions (3.1) and a part representing the fluctuations around the fixed background as

$$
A_{\mu}(x)=A_{\mu}^{(0)}(x)+\mathcal{A}_{\mu}(x)
$$

where $\mathcal{A}_{\mu}(x)$ satisfies the constraints

$$
\mathcal{A}_{\mu}\left(x+\Sigma_{\nu a} \hat{\nu}\right)=\Omega_{a}(x) \star \mathcal{A}_{\mu}(x) \star \Omega_{a}(x)^{\dagger} .
$$

This means that the field $\mathcal{A}_{\mu}(x)$ is an adjoint section of the corresponding gauge bundle over the noncommutative torus.

Let us now consider the noncommutative Yang-Mills action

$$
S=\frac{1}{g^{2}} \int \mathrm{d}^{D} x \operatorname{tr}_{(p)}\left(F_{\mu \nu}(x)-f_{\mu \nu} \mathbb{1}_{p}\right)_{\star}^{2}
$$


where the noncommutative field strength tensor $F_{\mu \nu}(x)$ is defined in (2.20). The constant background tensor field $f_{\mu \nu}$ will be specified later, and the integration is taken over the torus $\mathbb{T}^{D}$. Using the decomposition (3.8), the action (3.10) can be written as

$$
S=\frac{1}{g^{2}} \int \mathrm{d}^{D} x \operatorname{tr}(p)\left(\mathcal{F}_{\mu \nu}(x)+F_{\mu \nu}^{(0)}-f_{\mu \nu} \mathbb{1}_{p}\right)_{\star}^{2},
$$

where

$$
\begin{aligned}
\mathcal{F}_{\mu \nu}= & \partial_{\mu} \mathcal{A}_{\nu}-\partial_{\nu} \mathcal{A}_{\mu}+i\left(\mathcal{A}_{\mu} \star \mathcal{A}_{\nu}-\mathcal{A}_{\nu} \star \mathcal{A}_{\mu}\right) \\
& +i\left(A_{\mu}^{(0)} \star \mathcal{A}_{\nu}-\mathcal{A}_{\nu} \star A_{\mu}^{(0)}\right)-i\left(A_{\nu}^{(0)} \star \mathcal{A}_{\mu}-\mathcal{A}_{\mu} \star A_{\nu}^{(0)}\right) \\
F_{\mu \nu}^{(0)}= & \partial_{\mu} A_{\nu}^{(0)}-\partial_{\nu} A_{\mu}^{(0)}+i\left(A_{\mu}^{(0)} \star A_{\nu}^{(0)}-A_{\nu}^{(0)} \star A_{\mu}^{(0)}\right)=\left(F+\frac{1}{4} F \theta F\right)_{\mu \nu} \otimes \mathbb{1}_{p} .
\end{aligned}
$$

From (3.7) and the identity

$$
\left(\frac{1}{\mathbb{1}_{D}-\theta \alpha^{\top} \Sigma^{-1}}\right)^{2} \otimes \mathbb{1}_{p}=\left(\mathbb{1}_{D}+\frac{1}{2} \theta F\right)^{2} \otimes \mathbb{1}_{p}=\mathbb{1}_{D} \otimes \mathbb{1}_{p}+\theta F^{(0)}
$$

it follows that the relation 3.5$)$ is equivalent to

$$
\Sigma^{\top} F^{(0)} \Sigma=2 \pi \frac{1}{p \mathbb{1}_{D}+(Q+\gamma) \Theta}(Q+\gamma) \otimes \mathbb{1}_{p}
$$

Eq. (3.15) gives the relationship between the central curvatures, the topological charges, and the 't Hooft fluxes of the gauge field configurations. Requiring that $\mathcal{A}_{\mu}(x)=0$ be the vacuum field configuration of the theory up to a gauge transformation fixes $f_{\mu \nu} \mathbb{1}_{p}=F_{\mu \nu}^{(0)}$, so that the action becomes

$$
S=\frac{1}{g^{2}} \int \mathrm{d}^{D} x \operatorname{tr}(p)\left(\mathcal{F}_{\mu \nu}(x) \star \mathcal{F}_{\mu \nu}(x)\right) .
$$

\subsection{Transformation of the action}

In this subsection, we will derive the Morita equivalence relation between noncommutative Yang-Mills theories in arbitrary even dimension $D=2 d$. Specifically, we will show that the theory (3.10) with the constraint (3.1) is equivalent to another noncommutative gauge theory on a torus with single-valued gauge fields. For this, we first rewrite the gauge theory of the previous subsection in terms of Weyl operators. We introduce the hermitian operator $\hat{A}_{\mu}$ as in (2.17), and define the constant abelian background $\hat{A}_{\mu}^{(0)}$ and the transition function $\hat{\Omega}_{a}$ similarly. The explicit gauge choice $(3.2)$ corresponds to the unitary operator

$$
\hat{\Omega}_{a}=\mathrm{e}^{i \alpha_{a \mu} \hat{x}_{\mu}} \otimes \Gamma_{a}
$$


and, corresponding to (3.8), we decompose $\hat{A}_{\mu}$ as

$$
\hat{A}_{\mu}=\hat{A}_{\mu}^{(0)}+\hat{\mathcal{A}}_{\mu}
$$

The action (3.10) or (3.16) can be written as

$$
\begin{aligned}
S & =\frac{1}{g^{2}} \operatorname{Tr} \operatorname{tr}(p)\left(\left[\hat{\partial}_{\mu}, \hat{A}_{\nu}\right]-\left[\hat{\partial}_{\nu}, \hat{A}_{\mu}\right]+i\left[\hat{A}_{\mu}, \hat{A}_{\nu}\right]-f_{\mu \nu} \mathbb{1}_{p}\right)^{2} \\
& =\frac{1}{g^{2}} \operatorname{Tr} \operatorname{tr}\left(\left[\hat{\nabla}_{\mu}^{(0)}, \hat{\mathcal{A}}_{\nu}\right]-\left[\hat{\nabla}_{\nu}^{(0)}, \hat{\mathcal{A}}_{\mu}\right]+i\left[\hat{\mathcal{A}}_{\mu}, \hat{\mathcal{A}}_{\nu}\right]\right)^{2}
\end{aligned}
$$

where $f_{\mu \nu}$ may be given in terms of $\hat{A}_{\mu}^{(0)}$ as

$$
f_{\mu \nu} \mathbb{1}_{p}=\left[\hat{\partial}_{\mu}, \hat{A}_{\nu}^{(0)}\right]-\left[\hat{\partial}_{\nu}, \hat{A}_{\mu}^{(0)}\right]+i\left[\hat{A}_{\mu}^{(0)}, \hat{A}_{\nu}^{(0)}\right]
$$

and we have introduced the fiducial constant curvature connection

$$
\hat{\nabla}_{\mu}^{(0)}=\hat{\partial}_{\mu}+i \hat{A}_{\mu}^{(0)}
$$

The constraint (3.1) and the equivalent one $(\sqrt{3.9})$ can be written in terms of operators as

$$
\begin{aligned}
\mathrm{e}^{\Sigma_{\nu a} \hat{\partial}_{\nu}} \hat{A}_{\mu} \mathrm{e}^{-\Sigma_{\nu a} \hat{\partial}_{\nu}} & =\hat{\Omega}_{a} \hat{A}_{\mu} \hat{\Omega}_{a}^{\dagger}-i \hat{\Omega}_{a}\left[\hat{\partial}_{\mu}, \hat{\Omega}_{a}^{\dagger}\right] \\
\mathrm{e}^{\Sigma_{\nu a} \hat{\partial}_{\nu}} \hat{\mathcal{A}}_{\mu} \mathrm{e}^{-\Sigma_{\nu a} \hat{\partial}_{\nu}} & =\hat{\Omega}_{a} \hat{\mathcal{A}}_{\mu} \hat{\Omega}_{a}^{\dagger} .
\end{aligned}
$$

Our next task is to solve this constraint for the gauge field configurations.

First of all, we see from (3.4) that the $\Gamma_{a}$ are twist eating solutions for $\mathrm{SU}(p)$. For generic rank $p$ and flux matrix $Q$, these matrices may be constructed as follows [39]. For this, it is convenient to use the discrete $\operatorname{SL}(D, \mathbb{Z})$ symmetry of $\mathbb{T}^{D}$ to transform the twist eaters into matrices $\Gamma_{b}^{\circ}$ through

$$
\Gamma_{a}=\prod_{b=1}^{D}\left(\Gamma_{b}^{\circ}\right)^{\Lambda_{b a}},
$$

where $\Lambda \in \mathrm{SL}(D, \mathbb{Z})$. The transformed twist eaters $\Gamma_{a}^{\circ}$ satisfy the commutation relations

$$
\Gamma_{a}^{\circ} \Gamma_{b}^{\circ}=\mathrm{e}^{2 \pi i Q_{a b}^{\circ} / p} \Gamma_{b}^{\circ} \Gamma_{a}^{\circ}
$$

with $Q=\Lambda^{\top} Q^{\circ} \Lambda$. We can now choose $\Lambda$ so that the matrix $Q^{\circ}$ takes a canonical skewdiagonal form

$$
Q^{\circ}=\left(\begin{array}{ccccc}
0 & -q_{1} & & & \\
q_{1} & 0 & & & \\
& & \ddots & & \\
& & & 0 & -q_{d} \\
& & & q_{d} & 0
\end{array}\right) .
$$


Given the $d$ independent fluxes $q_{i} \in \mathbb{Z}$, we introduce the three integers

$$
p_{i}=\operatorname{gcd}\left(q_{i}, p\right) \quad, \quad \tilde{p}_{i}=\frac{p}{p_{i}} \quad, \quad \tilde{q}_{i}=\frac{q_{i}}{p_{i}}
$$

By construction, $\tilde{p}_{i}$ and $\tilde{q}_{i}$ are co-prime. A necessary and sufficient condition for the existence of solutions to (3.26) is 39 that the integer $\tilde{p}_{1} \cdots \tilde{p}_{d}$, which is the dimension of the irreducible representation of the Weyl-'t Hooft algebra (3.4), divides the rank $p$. This is a condition which must be met by the geometrical parameters of the given constant curvature bundle. In that case we write

$$
p=\tilde{p}_{0} \prod_{i=1}^{d} \tilde{p}_{i}
$$

and the twist eating solutions may then be given on the $\operatorname{subgroup} \mathrm{SU}\left(\tilde{p}_{1}\right) \otimes \cdots \otimes \mathrm{SU}\left(\tilde{p}_{d}\right) \otimes$ $\mathrm{SU}\left(\tilde{p}_{0}\right)$ of $\mathrm{SU}(p)$ as

$$
\begin{aligned}
\Gamma_{2 i-1}^{\circ} & =\mathbb{1}_{\tilde{p}_{1}} \otimes \cdots \otimes V_{\tilde{p}_{i}} \otimes \cdots \otimes \mathbb{1}_{\tilde{p}_{d}} \otimes \mathbb{1}_{\tilde{p}_{0}} \\
\Gamma_{2 i}^{\circ} & =\mathbb{1}_{\tilde{p}_{1}} \otimes \cdots \otimes\left(W_{\tilde{p}_{i}}\right)^{\tilde{q}_{i}} \otimes \cdots \otimes \mathbb{1}_{\tilde{p}_{d}} \otimes \mathbb{1}_{\tilde{p}_{0}}
\end{aligned}
$$

for $i=1, \ldots, d$. Here $V_{p}$ and $W_{p}$ are the $\mathrm{SU}(p)$ shift and clock matrices

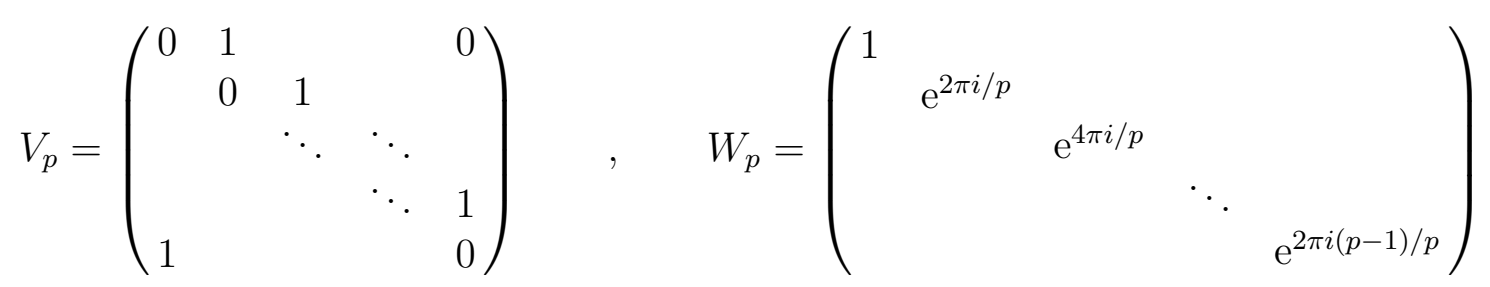

obeying $V_{p} W_{p}=\mathrm{e}^{2 \pi i / p} W_{p} V_{p}$. We can then obtain twist eaters $\Gamma_{a}$ satisfying (3.4) by using the relation (3.25). Note that the space of matrices which commute with the $\Gamma_{a}$ is the $\operatorname{gl}\left(\tilde{p}_{0}, \mathbb{C}\right)$ subspace of $\operatorname{gl}(p, \mathbb{C})$ generated by the matrices $\mathbb{1}_{\tilde{p}_{1}} \otimes \cdots \otimes \mathbb{1}_{\tilde{p}_{d}} \otimes Z_{0}, Z_{0} \in \operatorname{gl}\left(\tilde{p}_{0}, \mathbb{C}\right)$.

Since the set $\left\{\left(V_{p}\right)^{j}\left(W_{p}\right)^{j^{\prime}} \mid j, j^{\prime} \in \mathbb{Z}_{p}\right\}$ spans the linear space $\operatorname{gl}(p, \mathbb{C})$, the operator $\hat{\mathcal{A}}_{\mu}$ may be expanded as

$$
\hat{\mathcal{A}}_{\mu}=\sum_{\vec{j}} \int \mathrm{d}^{D} k \mathrm{e}^{i k_{\nu} \hat{x}_{\nu}} \otimes \prod_{a=1}^{D}\left(\Gamma_{a}\right)^{j_{a}} \otimes a_{\mu}(k, \vec{j})
$$

where the $\tilde{p}_{0} \times \tilde{p}_{0}$ matrix-valued coefficients $a_{\mu}(k, \vec{j})$ are periodic functions of $\vec{j}$ with the periodicity $j_{a} \sim j_{a}+\tilde{P}_{a b}, b=1, \ldots, D$. The matrix $\tilde{P}$ will be specified below. The constraint (3.24) then implies that $a_{\mu}(k, \vec{j})$ vanishes unless

$$
\zeta_{a}+\frac{1}{p} Q_{a b} j_{b}=n_{a} \in \mathbb{Z}
$$


where we have defined the $D$-dimensional real-valued vector

$$
\zeta_{a}=\frac{1}{2 \pi} k_{\nu}\left(\Sigma+\theta \alpha^{\top}\right)_{\nu a} .
$$

We will now find the general solution $\left(\zeta_{a}, j_{b}\right)$ to eq. (3.33). Introducing the $D \times D$ integral matrices

$$
\tilde{P}^{\circ}=\left(\begin{array}{ccccc}
\tilde{p}_{1} & 0 & & & \\
0 & \tilde{p}_{1} & & & \\
& & \ddots & & \\
& & & \tilde{p}_{d} & 0 \\
& & & 0 & \tilde{p}_{d}
\end{array}\right) \quad, \quad \tilde{Q}^{\circ}=\left(\begin{array}{ccccc}
0 & -\tilde{q}_{1} & & & \\
\tilde{q}_{1} & 0 & & & \\
& & \ddots & & \\
& & 0 & -\tilde{q}_{d} \\
& & & \tilde{q}_{d} & 0
\end{array}\right),
$$

and $\tilde{P}=\Lambda^{-1} \tilde{P}^{\circ} \Lambda^{\prime}, \tilde{Q}=\Lambda^{\top} \tilde{Q}^{\circ} \Lambda^{\prime}$, we can write

$$
Q=p \tilde{Q} \tilde{P}^{-1},
$$

where $\Lambda^{\prime} \in \mathrm{SL}(D, \mathbb{Z})$ will represent the automorphism symmetry group of the resulting theory that we shall find. Then eq. (3.33) can be written as $\zeta_{a}=m_{c}\left(\tilde{P}^{-1}\right)_{c a}$, where $m_{c}$ is a $D$-dimensional integral vector which satisfies

$$
m_{c}=n_{b} \tilde{P}_{b c}+j_{b} \tilde{Q}_{b c} .
$$

We will next show that for any given integer $m_{c}$, there exists a set of integers $\left(n_{b}, j_{b}\right)$ which satisfies (3.37). For this, we note that since $\tilde{p}_{i}$ and $\tilde{q}_{i}$ are relatively prime, there exists a set of integers $\left(a_{i}, b_{i}\right)$ such that

$$
a_{i} \tilde{p}_{i}+b_{i} \tilde{q}_{i}=1 \quad, \quad i=1, \ldots, d .
$$

Introducing the $D \times D$ integral matrices

$$
A^{\circ}=\left(\begin{array}{ccccc}
a_{1} & 0 & & & \\
0 & a_{1} & & & \\
& & \ddots & & \\
& & & a_{d} & 0 \\
& & & 0 & a_{d}
\end{array}\right) \quad, \quad B^{\circ}=\left(\begin{array}{ccccc}
0 & -b_{1} & & & \\
b_{1} & 0 & & & \\
& & \ddots & & \\
& & 0 & -b_{d} \\
& & & b_{d} & 0
\end{array}\right)
$$

and $A=\left(\Lambda^{\prime}\right)^{-1} A^{\circ} \Lambda, B=\left(\Lambda^{\prime}\right)^{-1} B^{\circ}\left(\Lambda^{-1}\right)^{\top}$, we have

$$
A \tilde{P}+B \tilde{Q}=\mathbb{1}_{D} .
$$

The integers $n_{b}=m_{a} A_{a b}$ and $j_{b}=m_{a} B_{a b}$ then give a solution to (3.37). It is easy to see that for any given $m_{a}$, the $j_{b}$ satisfying (3.37) are unique up to their periodicity. Thus, we find that the general solution to eq. (3.33) can be given by

$$
\zeta_{a}=m_{b}\left(\tilde{P}^{-1}\right)_{b a}, \quad j_{a}=m_{b} B_{b a} \quad \forall m_{b} \in \mathbb{Z} .
$$


Solving (3.34) for the momenta we obtain $k_{\mu}=2 \pi m_{a} \beta_{a \mu}$, where we have defined the $D \times D$ matrix

$$
\beta=\frac{1}{\left(\Sigma+\theta \alpha^{\top}\right) \tilde{P}}
$$

Therefore, the most general gauge field configuration satisfying the constraint (3.24) is given as

$$
\hat{\mathcal{A}}_{\mu}=\sum_{\vec{m} \in \mathbb{Z}^{D}} \mathrm{e}^{\pi i \sum_{a<b} \Theta_{a b}^{\prime} m_{a} m_{b}}\left(\prod_{a=1}^{D}\left(\hat{Z}_{a}^{\prime}\right)^{m_{a}}\right) \otimes \tilde{a}_{\mu}(\vec{m}),
$$

where $\tilde{a}_{\mu}(\vec{m})$ are $\tilde{p}_{0} \times \tilde{p}_{0}$ matrix-valued coefficients and

$$
\hat{Z}_{a}^{\prime}=\mathrm{e}^{2 \pi i \beta_{a \mu} \hat{x}_{\mu}} \otimes \prod_{b=1}^{D}\left(\Gamma_{b}\right)^{B_{a b}}
$$

Hermiticity of $\hat{\mathcal{A}}_{\mu}$ requires $\tilde{a}_{\mu}(-\vec{m})=\tilde{a}_{\mu}(\vec{m})^{\dagger}$. The operators 3.44) obey the commutation relations

$$
\begin{aligned}
\hat{Z}_{a}^{\prime} \hat{Z}_{b}^{\prime} & =\mathrm{e}^{-2 \pi i \Theta_{a b}^{\prime}} \hat{Z}_{b}^{\prime} \hat{Z}_{a}^{\prime} \\
{\left[\hat{\nabla}_{\mu}^{(0)}, \hat{Z}_{a}^{\prime}\right] } & =2 \pi i\left(\Sigma^{\prime-1}\right)_{a \mu} \hat{Z}_{a}^{\prime}
\end{aligned}
$$

where

$$
\begin{aligned}
\Theta^{\prime} & =\frac{1}{\Theta\left(\tilde{Q}+\frac{1}{p} \gamma \tilde{P}\right)-\tilde{P}}\left[\left(A-\frac{1}{p} B \gamma\right) \Theta+B\right]^{\top}, \\
\Sigma^{\prime} & =\Sigma\left[\Theta\left(\tilde{Q}+\frac{1}{p} \gamma \tilde{P}\right)-\tilde{P}\right] .
\end{aligned}
$$

The commutation relations $(3.45)$ are the same as (2.42) with the replacements $\hat{Z} \rightarrow \hat{Z}^{\prime}, \hat{\partial} \rightarrow$ $\hat{\nabla}^{(0)}, \Theta \rightarrow \Theta^{\prime}$ and $\Sigma \rightarrow \Sigma^{\prime}$. We may therefore define a basis $\hat{\Delta}^{\prime}\left(x^{\prime}\right)$ for the mapping of fields into operators as in (2.40). The $x_{\mu}^{\prime} \in \mathbb{R}$ are interpreted as coordinates on a new, dual torus with period matrix $\Sigma^{\prime}$ given by (3.47). Furthermore, the dimensionful noncommutativity parameters $\theta_{\mu \nu}^{\prime}$ which define the star-product are given by the dimensionless parameters (3.46) as

$$
\theta^{\prime}=\frac{1}{2 \pi} \Sigma^{\prime} \Theta^{\prime} \Sigma^{\prime \top}
$$

The expansion (3.43) then becomes

$$
\hat{\mathcal{A}}_{\mu}=\int \mathrm{d}^{D} x^{\prime} \hat{\Delta}^{\prime}\left(x^{\prime}\right) \otimes \mathcal{A}_{\mu}^{\prime}\left(x^{\prime}\right)
$$

where $\mathcal{A}_{\mu}^{\prime}\left(x^{\prime}\right)$ can be regarded as a single-valued $\mathrm{U}\left(\tilde{p}_{0}\right)$ gauge field on the noncommutative torus $\mathbb{T}_{\Theta^{\prime}}^{D}$ of total volume $\left|\operatorname{det} \Sigma^{\prime}\right|$. The operator trace $\operatorname{Tr}^{\prime}$, with $\operatorname{Tr}^{\prime} \hat{\Delta}^{\prime}\left(x^{\prime}\right)=1$, over the 
coordinates of this new torus is related to the original trace $\operatorname{Tr}$ through

$$
\operatorname{Tr}^{\prime} \operatorname{tr}\left(\tilde{p}_{0}\right)=\frac{\tilde{p}_{0}}{p}\left|\frac{\operatorname{det} \Sigma^{\prime}}{\operatorname{det} \Sigma}\right| \operatorname{Tr} \operatorname{tr}(p) .
$$

Using (3.20) and (3.50) we arrive at the canonical form of the noncommutative Yang-Mills action for the gauge field in (3.49),

$$
S=\frac{1}{g^{\prime 2}} \int \mathrm{d}^{D} x^{\prime} \operatorname{tr}_{\left(\tilde{p}_{0}\right)}\left(\mathcal{F}_{\mu \nu}^{\prime}\left(x^{\prime}\right) \star^{\prime} \mathcal{F}_{\mu \nu}\left(x^{\prime}\right)\right)
$$

where

$$
\mathcal{F}_{\mu \nu}^{\prime}=\partial_{\mu}^{\prime} \mathcal{A}_{\nu}^{\prime}-\partial_{\nu}^{\prime} \mathcal{A}_{\mu}^{\prime}+i\left(\mathcal{A}_{\mu}^{\prime} \star^{\prime} \mathcal{A}_{\nu}^{\prime}-\mathcal{A}_{\nu}^{\prime} \star^{\prime} \mathcal{A}_{\mu}^{\prime}\right)
$$

$\star^{\prime}$ denotes the star product defined using $\theta^{\prime}$ instead of $\theta$, and the new Yang-Mills coupling constant is given by

$$
g^{\prime 2}=g^{2} \frac{\left|\operatorname{det}\left[\Theta(Q+\gamma)-p \mathbb{1}_{D}\right]\right|}{\tilde{p}_{0} p^{D-1}},
$$

where we have used (3.36).

We have therefore shown that the $\mathrm{U}(p)$ gauge theory (3.10) on a bundle of topological charges $Q_{a b}$ and 't Hooft fluxes $\gamma_{a b}$ over the noncommutative torus $\mathbb{T}_{\Theta}^{D}$ (i.e. with gauge fields obeying twisted boundary conditions) is equivalent to the $\mathrm{U}\left(\tilde{p}_{0}\right)$ gauge theory (3.51) on a trivial bundle over the noncommutative torus $\mathbb{T}_{\Theta^{\prime}}^{D}$ (i.e. with gauge fields obeying periodic boundary conditions) with noncommutativity parameter matrix defined by (3.46) and the reduced rank $\tilde{p}_{0}$ by (3.29). In particular, for $\tilde{p}_{0}=1$, the internal matrix structure of the gauge fields are completely absorbed into the operators $\hat{Z}_{a}^{\prime}$ which are regarded as the coordinate generators of $\mathbb{T}_{\Theta^{\prime}}^{D}$, thereby allowing one to reinterpret the original non-abelian gauge theory as a $U(1)$ gauge theory on the noncommutative torus $\mathbb{T}_{\Theta^{\prime}}^{D}$ (Note that this statement is true even for $\theta=0)$. On the other hand, when $Q=0$ we have $\tilde{p}_{0}=p$ and the rank of the gauge fields is unchanged. Note that from (3.46) and (3.47) it follows that, when $\theta=0$, the resulting dual gauge theory does not depend on $\gamma$ and $\alpha$ separately, but only on their combination given by $Q$ in (3.5). This means that keeping both $\gamma$ and $\alpha$ non-zero is in fact redundant in the commutative case. But for $\theta \neq 0$, this is not quite the case.

\footnotetext{
${ }^{3}$ The transformation (3.53) of the Yang-Mills coupling constant differs from the standard one which is derived using string theoretical T-duality. The source of this discrepency owes to the normalization of the trace $\operatorname{Tr}$ on the noncommutative torus $\mathbb{T}_{\Theta}^{D}$. In string theory, this trace is normalized according to the standard formula in noncommutative geometry for the rank of the module of sections of the fundamental bundle associated to the given Chan-Paton gauge bundle over $\mathbb{T}_{\Theta}^{D}$ (This module coincides with the Hilbert space of open string ground states) [10, 33. In the present field theoretical case, which does not require the specification of any representation of the Weyl operators, we have chosen the more natural volume normalization $\operatorname{Tr} \mathbb{1}=|\operatorname{det} \Sigma|$.
} 
We note that eq. (3.40) and the antisymmetry of the matrices $A B^{\top}$ and $\tilde{Q}^{\top} \tilde{P}$ implies the matrix identity

$$
\left(\begin{array}{cc}
A & B \\
\tilde{Q}^{\top} & -\tilde{P}^{\top}
\end{array}\right)\left(\begin{array}{cc}
0 & \mathbb{1}_{D} \\
\mathbb{1}_{D} & 0
\end{array}\right)\left(\begin{array}{cc}
A & B \\
\tilde{Q}^{\top} & -\tilde{P}^{\top}
\end{array}\right)^{\top}=\left(\begin{array}{cc}
0 & \mathbb{1}_{D} \\
\mathbb{1}_{D} & 0
\end{array}\right) .
$$

It follows that, when $\gamma=0$, the map $\Theta \mapsto \Theta^{\prime}$ in $(3.46)$ is the usual $\operatorname{SO}(D, D ; \mathbb{Z})$ transformation that relates Morita equivalent noncommutative tori [33]. From the present point of view, Morita equivalence is therefore regarded as a change of basis $\hat{\Delta}(x) \leftrightarrow \hat{\Delta}^{\prime}\left(x^{\prime}\right)$ for the mapping between Weyl operators and fields. The choice $\gamma=0$ is required whenever

one wants to represent the operators $\hat{Z}_{a}^{\prime}$ on the Hilbert space of sections of the associated fundamental bundle, as in the case of D-brane applications wherein the open string wavefunctions transform in the fundamental representation of the Chan-Paton gauge group [10]. A choice $\gamma \neq 0$ is possible for representations on the Hilbert space of sections of the associated principal bundle. Notice also that for $d>1$ the calculation presented above accounts for only a subset of the possible noncommutative gauge theories, since in that instance, even in the commutative case, generic bundles over the torus do not always admit constant curvature connections such as (3.22). However, the derivation above does allow for arbitrary non-abelian 't Hooft fluxes $\gamma$.

\subsection{Star-gauge symmetry and transformation of observables}

We will now demonstrate explicitly how the star-gauge symmetries of two Morita equivalent Yang-Mills theories, as well as their star-gauge invariant observables, are related to each other. We begin with the $\mathrm{U}\left(\tilde{p}_{0}\right)$ theory (3.20) in which all fields obey periodic boundary conditions. Its gauge symmetry is

$$
\hat{\mathcal{A}}_{\mu} \mapsto \hat{g} \hat{\mathcal{A}}_{\mu} \hat{g}^{\dagger}-i \hat{g}\left[\hat{\nabla}_{\mu}^{(0)}, \hat{g}^{\dagger}\right]
$$

where $\hat{g}$ may be written as

$$
\hat{g}=\int \mathrm{d}^{D} x^{\prime} \hat{\Delta}^{\prime}\left(x^{\prime}\right) \otimes g^{\prime}\left(x^{\prime}\right)
$$

The gauge function $g^{\prime}\left(x^{\prime}\right)$ is a single-valued, $\tilde{p}_{0} \times \tilde{p}_{0}$ star $\left(\star^{\prime}\right)$-unitary matrix field, which parametrizes the usual gauge transformation of the noncommutative Yang-Mills theory (3.51). To see how this is interpreted as the gauge symmetry of the $\mathrm{U}(p)$ theory (3.19), we use (3.22) to rewrite (3.55) as

$$
\hat{A}_{\mu} \mapsto \hat{g} \hat{A}_{\mu} \hat{g}^{\dagger}-i \hat{g}\left[\hat{\partial}_{\mu}, \hat{g}^{\dagger}\right],
$$

where $\hat{A}_{\mu}$ is given by (3.18). The invariance of the action (3.19) under (3.57) follows by construction. Note that by the definition (3.56), the operator $\hat{g}$ obeys

$$
\mathrm{e}^{\Sigma_{\mu a} \hat{\partial}_{\mu}} \hat{g} \mathrm{e}^{-\Sigma_{\mu a} \hat{\partial}_{\mu}}=\hat{\Omega}_{a} \hat{g} \hat{\Omega}_{a}^{\dagger}
$$


from which it follows that the gauge transformed operator in (3.57) also satisfies the required twisted boundary condition (3.23). Let us rewrite (3.58) in terms of fields on the noncommutative torus $\mathbb{T}_{\Theta}^{D}$. For this, we expand the Weyl operator $\hat{g}$ using the basis $\hat{\Delta}(x)$ as

$$
\hat{g}=\int \mathrm{d}^{D} x \hat{\Delta}(x) \otimes g(x)
$$

Due to (3.58), the $p \times p$ star( $\star$ )-unitary matrix field $g(x)$ is multi-valued and defines an adjoint section of the gauge bundle,

$$
g\left(x+\Sigma_{\mu a} \hat{\mu}\right)=\Omega_{a}(x) \star g(x) \star \Omega_{a}(x)^{\dagger} .
$$

Let us now consider $\operatorname{star}\left(\star^{\prime}\right)$-gauge invariant observables in the $\mathrm{U}\left(\tilde{p}_{0}\right)$ theory. Since the fields of this theory are single-valued functions on the torus, we may use the construction of Sections 2.3 and 2.4. Given the parallel transport operators

$$
\mathcal{U}^{\prime}\left(x^{\prime} ; C\right)=\mathrm{P} \exp _{\star^{\prime}}\left(i \int_{C} \mathrm{~d} \xi^{\mu} \mathcal{A}_{\mu}^{\prime}\left(x^{\prime}+\xi\right)\right)
$$

where notation is as in Section [2.3, we may define a star $\left(\star^{\prime}\right)$-gauge invariant observable by

$$
\mathcal{O}(C)=\int \mathrm{d}^{D} x^{\prime} \operatorname{tr}_{\left(\tilde{p}_{0}\right)}\left(\mathcal{U}^{\prime}\left(x^{\prime} ; C\right) \star^{\prime} S_{v}^{\prime}\left(x^{\prime}\right)\right)
$$

where

$$
S_{v}^{\prime}\left(x^{\prime}\right)=\mathrm{e}^{i k_{\mu}^{\prime} x_{\mu}^{\prime}} \mathbb{1}_{\tilde{p}_{0}} .
$$

The total momentum $k_{\mu}^{\prime}$ is quantized as

$$
k_{\mu}^{\prime}=2 \pi\left(\Sigma^{\prime}-1\right)_{a \mu} m_{a}^{\prime}
$$

while the constraint on the relative separation vector between the two ends of the contour $C$ is

$$
v_{\mu}=\theta_{\mu \nu}^{\prime} k_{\nu}^{\prime}+\Sigma_{\mu a}^{\prime} n_{a}^{\prime}
$$

for some integer-valued vectors $m_{a}^{\prime}$ and $n_{a}^{\prime}$. We now rewrite the above observables in terms of Weyl operators. Introducing

$$
\begin{aligned}
& \hat{U}^{\prime}(C)=\mathrm{P} \exp \left(\int_{C} \mathrm{~d} \xi^{\mu}\left(\hat{\nabla}_{\mu}^{(0)}+i \hat{\mathcal{A}}_{\mu}\right)\right), \\
& \hat{D}^{\prime}(C)=\mathrm{P} \exp \left(\int_{C} \mathrm{~d} \xi^{\mu} \hat{\nabla}_{\mu}^{(0)}\right),
\end{aligned}
$$


we can define a star $\left(\star^{\prime}\right)$-gauge invariant observable as in (2.37) by

$$
\mathcal{O}(C)=\operatorname{Tr}^{\prime} \operatorname{tr}_{\left(\tilde{p}_{0}\right)}\left(\hat{U}^{\prime}(C) \hat{D}^{\prime}(C)^{\dagger} \hat{S}_{v}\right)
$$

where the unitary operator $\hat{S}_{v}$ may be expanded as

$$
\hat{S}_{v}=\int \mathrm{d}^{D} x^{\prime} \hat{\Delta}^{\prime}\left(x^{\prime}\right) \otimes S_{v}^{\prime}\left(x^{\prime}\right)
$$

and it satisfies

$$
\hat{S}_{v} \hat{\Delta}^{\prime}\left(x^{\prime}\right) \hat{S}_{v}^{\dagger}=\hat{\Delta}^{\prime}\left(x^{\prime}+v\right) \otimes \mathbb{1}_{\tilde{p}_{0}}
$$

Let us now see how to interpret the operator (3.67) as a sensible quantity also in the Morita equivalent $\mathrm{U}(p)$ theory. We first define a parallel transport operator $\mathcal{U}(x ; C)$ in the $\mathrm{U}(p)$ theory using Eqs. (2.31) and (2.32). Note that $\hat{U}(C)=\hat{U}^{\prime}(C)$. The observable defined in (3.67) can then be rewritten as

$$
\mathcal{O}(C)=\operatorname{Tr} \operatorname{tr}_{(p)}\left[\left(\hat{U}(C) \hat{D}(C)^{\dagger}\right)\left(\hat{D}(C) \hat{D}^{\prime}(C)^{\dagger}\right) \hat{S}_{v}\right] .
$$

We now expand the operator $\hat{S}_{v}$ in terms of the basis $\hat{\Delta}(x)$ similarly to (3.59) and define the $p \times p \operatorname{star}(\star)$-unitary matrix field $S_{v}(x)$ as an adjoint section. Then, using (2.31), (3.66) and (3.22), we can write the quantity (3.70) as

$$
\mathcal{O}(C)=\int \mathrm{d}^{D} x \operatorname{tr}_{(p)}\left(\mathcal{U}(x ; C) \star S_{v}^{(0)}(x)\right),
$$

where we have defined the $p \times p$ star $(\star)$-unitary matrix field

$$
S_{v}^{(0)}(x)=\left[\mathrm{P} \exp _{\star}\left(i \int_{C} \mathrm{~d} \xi^{\mu} A_{\mu}^{(0)}(x+\xi)\right)\right]^{*} \star S_{v}(x) .
$$

By construction, (3.71) is star( $($ )-gauge invariant, which can also be checked by recalling the $\operatorname{star}(\star)$-gauge transformation (2.29) of the parallel transport operator $\mathcal{U}(x ; C)$ and the translation-generating property of the field (3.72),

$$
S_{v}^{(0)}(x) \star g(x) \star S_{v}^{(0)}(x)^{\dagger}=g(x+v)
$$

where $g(x)$ is an arbitrary adjoint section as in (3.60). Note that the integrand of (3.71) is a single-valued function because of the boundary conditions

$$
\begin{aligned}
\mathcal{U}\left(x+\Sigma_{\mu a} \hat{\mu} ; C\right) & =\Omega_{a}(x) \star \mathcal{U}(x ; C) \star \Omega_{a}(x+v)^{\dagger}, \\
S_{v}^{(0)}\left(x+\Sigma_{\mu a} \hat{\mu}\right) & =\Omega_{a}(x+v) \star S_{v}^{(0)}(x) \star \Omega_{a}(x)^{\dagger}
\end{aligned}
$$

where the relation (3.75) is required for consistency of the equations (3.73) and (3.60). 
It follows that $\operatorname{star}\left(\star^{\prime}\right)$-gauge invariant observables of the $\mathrm{U}\left(\tilde{p}_{0}\right)$ theory can be interpreted as star $(\star)$-gauge invariant observables of the $\mathrm{U}(p)$ theory. As in Section 2.3, we can show that the field $S_{v}^{(0)}(x)$ satisfying (3.73) on the noncommutative torus $\mathbb{T}_{\Theta}^{D}$ is in fact unique, so that the Morita equivalence relation provides a one-to-one mapping between the quantum correlation functions of the two noncommutative Yang-Mills theories. An instructive example which shows how this correspondence works is the $\theta=0$ case. Then, one finds that Polyakov lines of commutative gauge theory map to open loops in the Morita equivalent noncommutative gauge theory.

The observables (3.71, 3.72) are the appropriate modifications of the observables constructed in Sections 2.3 and 2.4 to the case of multi-valued gauge fields on the noncommutative torus. We remark that the observables constructed here using the formalism of Weyl operators differ from the noncommutative holonomy operators constructed recently in 35] which are star-gauge invariant without the need of introducing the operator trace Tr, but which do not immediately generalize to gauge field configurations of non-vanishing magnetic flux. The present noncommutative Wilson lines are defined for multi-valued gauge fields, and moreover, as we will see in Section 6, they are the ones which arise in the effective actions for charges propagating in the background of noncommutative gauge fields.

\section{Lattice regularization}

In this Section we will consider a lattice regularization of noncommutative field theories. Through a general analysis of the noncommutative algebra generated by the spacetime coordinates, we will find that the discretization of the spacetime inevitably requires that it be compact in some restricted way depending on the noncommutativity. Due to this restriction, the commutative limit is not commutable with the continuum limit, which demonstrates the $\mathrm{UV} / \mathrm{IR}$ mixing property of noncommutative field theories at a fully nonperturbative level. We will show that the discrete formulation allows a nonperturbative regularization of generic noncommutative field theories with single-valued fields. Given the Morita equivalence property discussed in the previous Section, this means that noncommutative Yang-Mills theories with gauge fields obeying twisted boundary conditions can also be regularized in terms of the Morita equivalent Yang-Mills theories with fields satisfying periodic boundary conditions, insofar as defining regularized correlation functions of star-gauge invariant observables are concerned. In this way, we obtain a nonperturbative definition of noncommutative YangMills theories in arbitrary even dimensions with multi-valued or single-valued gauge fields. 


\subsection{General construction}

To describe the lattice regularization of noncommutative Yang-Mills theory, there are some subtle technical points concerning the transcription of the continuum theory onto a lattice that we first need to address. To this end, let us go back to the construction of Section 2.1. To define a lattice field theory, we restrict the spacetime points to $x_{\mu} \in \epsilon \mathbb{Z}$, where $\epsilon$ is the lattice spacing. It follows that the lattice momentum must be identified under the shift

$$
k_{\mu} \mapsto k_{\mu}+\frac{2 \pi}{\epsilon} \delta_{\mu \nu}, \quad \nu=1, \ldots, D
$$

Correspondingly, there is the operator identity

$$
\mathrm{e}^{i\left(k_{\mu}+\frac{2 \pi}{\epsilon} \delta_{\mu \nu}\right) \hat{x}_{\mu}}=\mathrm{e}^{i k_{\mu} \hat{x}_{\mu}}, \quad \nu=1, \ldots, D
$$

By acting with the operator $\mathrm{e}^{-i k_{\mu} \hat{x}_{\mu}}$ on both sides of (4.2), we find that there is also the operator identity

$$
\mathrm{e}^{2 \pi i \hat{x}_{\mu} / \epsilon}=\mathbb{1}, \quad \mu=1, \ldots, D
$$

and, moreover, that the momentum $k_{\mu}$ is quantized according to

$$
\theta_{\mu \nu} k_{\nu} \in 2 \epsilon \mathbb{Z}
$$

For $k_{\mu}$ satisfying (4.4), the commutation relation

$$
\mathrm{e}^{i k_{\mu} \hat{x}_{\mu}} \mathrm{e}^{2 \pi i \hat{x}_{\nu} / \epsilon}=\mathrm{e}^{2 \pi i \theta_{\mu \nu} k_{\mu} / \epsilon} \mathrm{e}^{2 \pi i \hat{x}_{\nu} / \epsilon} \mathrm{e}^{i k_{\mu} \hat{x}_{\mu}}, \quad \mu=1, \ldots, D
$$

is compatible with the identity (4.3). On the other hand, the compatibility of the commutation relation

$$
\mathrm{e}^{v_{\nu} \hat{\partial}_{\nu}} \mathrm{e}^{2 \pi i \hat{x}_{\mu} / \epsilon} \mathrm{e}^{-v_{\nu} \hat{\partial}_{\nu}}=\mathrm{e}^{2 \pi i\left(\hat{x}_{\mu}+v_{\mu}\right) / \epsilon}
$$

with the identity (4.3) requires $v_{\mu} \in \epsilon \mathbb{Z}$. Thus we are led to use instead the lattice shift operator

$$
\hat{D}_{\mu}=\mathrm{e}^{\epsilon \hat{\partial}_{\mu}}
$$

but not the derivation $\hat{\partial}_{\mu}$ itself anymore. The restriction to the lattice shift operator (4.7) is standard in lattice field theory, whereas the restriction (4.4) on the lattice momentum simply disappears in the commutative case $\theta_{\mu \nu}=0$ and is quite characteristic of the noncommutative geometry.

The momentum quantization (4.4) requires that the spacetime be compactified in some restricted way. Let us consider the case when the fields have the periodic boundary conditions

$$
\phi\left(x+\Sigma_{\mu a} \hat{\mu}\right)=\phi(x), \quad a=1, \ldots, D
$$


where the periods $\Sigma_{\mu a}$ are integer multiples of the lattice spacing $\epsilon$. Because of (4.8), the momentum is quantized as

$$
k_{\mu}=2 \pi\left(\Sigma^{-1}\right)_{a \mu} m_{a}, \quad m_{a} \in \mathbb{Z} .
$$

The momentum periodicity (4.1) due to the lattice discretization $x_{\mu} \in \epsilon \mathbb{Z}$ can be recast in terms of the integers $m_{a}$ as

$$
m_{a} \mapsto m_{a}+\frac{1}{\epsilon} \Sigma_{\mu a}, \quad \mu=1, \ldots, D
$$

Now the restriction on the momentum given by (4.4) implies that there exists a $D \times D$ integer-valued matrix $M_{\mu a}$ which satisfies

$$
M_{\mu a} \Sigma_{\nu a}=\frac{\pi}{\epsilon} \theta_{\mu \nu}
$$

We have therefore discovered the remarkable fact that lattice regularization of noncommutative field theory forces the spacetime to be compact. For fixed $M$, say $M=\mathbb{1}_{D}$, the infrared cutoff disappears as $\frac{1}{\epsilon}$ in the continuum limit $\epsilon \rightarrow 0$. Note that the commutative limit $\theta_{\mu \nu} \rightarrow 0$ does not commute with the continuum limit $\epsilon \rightarrow 0$. Thus the UV/IR mixing discovered in perturbative analyses of noncommutative field theories [16] is demonstrated here at a fully nonperturbative level. On the other hand, in order to obtain a continuum spacetime of finite volume one has to send $M \rightarrow \infty$ as one takes the $\epsilon \rightarrow 0$ limit. It is important to note that for any given period matrix $\Sigma_{\mu a}$ and noncommutativity parameter $\theta_{\mu \nu}$ in the continuum, one can construct a family of lattice geometries satisfying the restriction (4.11) and approaching the target continuum theory in the $\epsilon \rightarrow 0$ limit. This means that in spite of the restriction (4.11) for the regularized field theories, one can obtain the most general noncommutative geometries parametrized by $\Sigma_{\mu a}$ and $\theta_{\mu \nu}$ in the continuum limit.

Due to the momentum quantization (4.9), we are led to use the coordinate operators

$$
\hat{Z}_{a}=\mathrm{e}^{2 \pi i\left(\Sigma^{-1}\right)_{a \mu} \hat{x}_{\mu}}
$$

but not the $\hat{x}_{\mu}$ themselves anymore. The commutation relations of the operators (4.12) and (4.7) are

$$
\begin{aligned}
\hat{Z}_{a} \hat{Z}_{b} & =\mathrm{e}^{-2 \pi i \Theta_{a b}} \hat{Z}_{b} \hat{Z}_{a} \\
\hat{D}_{\mu} \hat{Z}_{a} \hat{D}_{\mu}^{\dagger} & =\mathrm{e}^{2 \pi i \epsilon\left(\Sigma^{-1}\right)_{a \mu}} \hat{Z}_{a}
\end{aligned}
$$

where the dimensionless noncommutativity parameter

$$
\Theta_{a b}=2 \pi\left(\Sigma^{-1}\right)_{a \mu} \theta_{\mu \nu}\left(\Sigma^{-1}\right)_{b \nu}
$$


is necessarily rational-valued on the lattice, since the restriction (4.11) implies that

$$
M_{\mu a}=\frac{1}{2 \epsilon} \Sigma_{\mu b} \Theta_{b a}
$$

is an integer-valued matrix. Because of (4.3), we also have the identity

$$
\prod_{a=1}^{D}\left(\hat{Z}_{a}\right)^{\frac{1}{\epsilon} \Sigma_{\mu a}}=\exp \left[\pi i \sum_{a<b} \Sigma_{\mu a} \Theta_{a b} \Sigma_{\mu b}\right] \cdot \mathbb{1}_{D}, \quad \mu=1, \ldots, D
$$

By construction, the identity (4.17) is compatible with the commutation relations (4.13) and (4.14).

We can define a map $\hat{\Delta}(x)$ between lattice fields and Weyl operators by

$$
\hat{\Delta}(x)=\frac{1}{\left|\operatorname{det} \frac{1}{\epsilon} \Sigma\right|} \sum_{\vec{m}}\left(\prod_{a=1}^{D}\left(\hat{Z}_{a}\right)^{m_{a}}\right) \mathrm{e}^{-\pi i \sum_{a<b} \Theta_{a b} m_{a} m_{b}} \mathrm{e}^{-2 \pi i\left(\Sigma^{-1}\right)_{a \mu} m_{a} x_{\mu}}
$$

where the sum goes over $\mathbb{Z}^{D}$ modulo the periodicity (4.10) and $x_{\mu}$ is a point on the spacetime lattice $\epsilon \mathbb{Z}$ with periodicity (4.8). Note that

$$
\frac{1}{\left|\operatorname{det} \frac{1}{\epsilon} \Sigma\right|} \sum_{\vec{m}} \mathrm{e}^{2 \pi i\left(\Sigma^{-1}\right)_{a \mu} m_{a} x_{\mu}}=\delta_{x, 0(\bmod \Sigma)} .
$$

As we did in (2.14), we can define the lattice star-product using the map $\hat{\Delta}(x)$ defined in (4.18). Explicitly it can be given as

$$
\phi_{1}(x) \star \phi_{2}(x)=\sum_{y, z} K(x-y, x-z) \phi_{1}(y) \phi_{2}(z)
$$

where the sums go over the spacetime lattice points $\epsilon \mathbb{Z}$ modulo the periodicity (4.8). The kernel $K$ in (4.20) is given by

$$
K(y, z)=\frac{1}{\left|\operatorname{det} \frac{1}{\epsilon} \Sigma\right|^{2}} \sum_{\vec{m}, \vec{n}} \exp \left[2 \pi i\left(\Sigma^{-1}\right)_{a \mu}\left(m_{a} y_{\mu}+n_{a} z_{\mu}\right)+i \pi \Theta_{a b} m_{a} n_{b}\right] .
$$

The map (4.18) and the star-product (4.20,4.21) possess all the algebraic properties that their continuum counterparts have. If $2 \epsilon(\Sigma \Theta)^{-1}=M^{-1}$ is an integer-valued matrix, where $M$ is the integral matrix introduced in (4.11), then the sums in (4.21) can be done explicitly yielding

$$
K(y, z)=\frac{1}{\left|\operatorname{det} \frac{1}{\epsilon} \Sigma\right|} \mathrm{e}^{-2 i\left(\theta^{-1}\right)_{\mu \nu} y_{\mu} z_{\nu}} .
$$

The corresponding formula (4.20) for the lattice star-product is then analogous to the second expression in (2.14) for the continuum star-product. 
Let us now turn to the construction of field theories on a discrete noncommutative torus. In the case of a scalar lattice field $\phi(x)$, one can define the operator

$$
\hat{\phi}=\sum_{x} \phi(x) \hat{\Delta}(x)
$$

where the sum runs over lattice points. We may then write down an action

$$
S[\hat{\phi}]=\operatorname{Tr}\left(\frac{1}{2} \sum_{\mu}\left(\hat{D}_{\mu} \hat{\phi} \hat{D}_{\mu}^{\dagger}-\hat{\phi}\right)^{2}+\frac{1}{2} \hat{\phi}^{2}+\frac{1}{4 !} \hat{\phi}^{4}\right)
$$

which leads to the usual lattice action of the field $\phi(x)$ with star-interaction term analogous to (2.16).

A huge advantage of the lattice regularization is that it allows one to construct finite dimensional representations of the noncommutative geometry. This is apparent already at the level of the lattice operators (4.18). It is straightforward to compute that they obey the commutation relations

$$
[\hat{\Delta}(x), \hat{\Delta}(y)]=\sum_{z} \mathcal{K}(x-z, y-z) \hat{\Delta}(z)
$$

where

$$
\mathcal{K}(x, y)=\frac{2 i}{\left|\operatorname{det} \frac{1}{\epsilon} \Sigma\right|^{2}} \sum_{\vec{m}, \vec{n}} \sin \left(\pi \Theta_{a b} m_{a} n_{b}\right) \mathrm{e}^{2 \pi i\left(\Sigma^{-1}\right)_{a \mu}\left(m_{a} x_{\mu}+n_{a} y_{\mu}\right)}
$$

The lattice operators $\hat{\Delta}(x)$ therefore generate a finite dimensional Lie algebra of dimension $\left|\operatorname{det} \frac{1}{\epsilon} \Sigma\right|=2^{D}|\operatorname{det} M / \operatorname{det} \Theta|$. In the lattice field theory, the operators $\hat{\Delta}(x)$ can in this way be regarded as maps from the $\left|\operatorname{det} \frac{1}{\epsilon} \Sigma\right|$ lattice points to a finite dimensional matrix representation, thereby illustrating how the degrees of freedom are mapped into each other in the one-to-one correspondence between Weyl operators and fields. Indeed, the algebra (4.13) can always be represented by finite dimensional matrices which admit a tensor product decomposition into blocks depending on the detailed forms of the rational numbers $\Theta_{a b}$ (See 22] for some specific examples). We shall return to this point in the next Section. The lattice regularization can therefore also be thought of as an approximation to the algebra of Weyl operators in the continuum by finite dimensional matrix algebras. This constitutes the standard description of noncommutative lattices by approximately finite algebras in noncommutative geometry [31]. The rigorous definition of the continuum limit described above within such an algebraic description of the noncommutative geometry is given in Ref. 40. 


\subsection{Noncommutative Yang-Mills theory on the lattice}

In order to construct a lattice regularization of noncommutative Yang-Mills theory, we need to maintain star-gauge invariance on the lattice. As in the case of ordinary lattice gauge theory [29], this is achieved by putting the gauge fields on the links of the lattice [22],

$$
\hat{U}_{\mu}=\sum_{x} \hat{\Delta}(x) \otimes U_{\mu}(x)
$$

where $\hat{U}_{\mu}$ is a unitary operator and $U_{\mu}(x)$ is a $p \times p$ matrix field on the lattice which is star-unitary,

$$
U_{\mu}(x) \star U_{\mu}(x)^{\dagger}=\mathbb{1}_{p}
$$

One can write an action

$$
S=-\frac{1}{g^{2}} \sum_{\mu \neq \nu} \operatorname{Tr} \operatorname{tr}(p)\left[\hat{U}_{\mu}\left(\hat{D}_{\mu} \hat{U}_{\nu} \hat{D}_{\mu}^{\dagger}\right)\left(\hat{D}_{\nu} \hat{U}_{\mu}^{\dagger} \hat{D}_{\nu}^{\dagger}\right) \hat{U}_{\nu}^{\dagger}\right]
$$

which is invariant under the transformation

$$
\hat{U}_{\mu} \mapsto \hat{g} \hat{U}_{\mu}\left(\hat{D}_{\mu} \hat{g}^{\dagger} \hat{D}_{\mu}^{\dagger}\right)
$$

The action (4.29) can be written in terms of the lattice fields $U_{\mu}(x)$ as

$$
S=-\frac{1}{g^{2}} \sum_{x} \sum_{\mu \neq \nu} \operatorname{tr}_{(p)}\left[U_{\mu}(x) \star U_{\nu}(x+\epsilon \hat{\mu}) \star U_{\mu}(x+\epsilon \hat{\nu})^{\dagger} \star U_{\nu}(x)^{\dagger}\right],
$$

which is invariant under the lattice star-gauge transformation

$$
U_{\mu}(x) \mapsto g(x) \star U_{\mu}(x) \star g(x+\epsilon \hat{\mu})^{\dagger},
$$

where the gauge function $g(x)$ is defined by

$$
\hat{g}=\sum_{x} \hat{\Delta}(x) \otimes g(x)
$$

and it is star-unitary, $g(x) \star g(x)^{\dagger}=\mathbb{1}_{p}$.

This discrete version of noncommutative Yang-Mills theory has several benefits. First of all, it provides a concrete definition of the quantum gauge theory path integral in the continuum. The unitary operators (4.27) live in a finite-dimensional operator algebra and are therefore elements of a compact unitary group. Note that the trace $\operatorname{Tr} \operatorname{tr}(p)$ which appears in $(4.29)$ corresponds to the trace in the fundamental representation of this unitary group. The lattice gauge theory path integral may then be defined by the measure $\mathrm{d} \hat{U}$ which is the Haar measure of this compact Lie group that is invariant under the left and right actions

$$
\hat{U}_{\mu} \mapsto \hat{g} \hat{U}_{\mu} \quad, \quad \hat{U}_{\mu} \mapsto \hat{U}_{\mu} \hat{g}
$$


In terms of the star-unitary lattice fields $U_{\mu}(x)$, the path integral measure $\mathcal{D} U_{\mu}(x)$ is given by the Haar measure of the compact Lie group which is formed by the $U_{\mu}(x)$ with multiplication given by the star-product, and which is invariant under the left and right actions $U_{\mu}(x) \mapsto$ $g(x) \star U_{\mu}(x)$ and $U_{\mu}(x) \mapsto U_{\mu}(x) \star g(x)$. This measure preserves star-gauge invariance. In the commutative limit $\theta_{\mu \nu}=0$, the discrete noncommutative Yang-Mills theory reduces to ordinary lattice gauge theory [29], including the path integral measure because of the uniqueness of the Haar measure. The lattice regularization also gives an approximation to the star-gauge symmetry group in the continuum. An algebraic description of this continuum gauge group is given in Ref. 41].

Let us now describe the discrete analogs of the star-gauge invariant observables introduced in Section 2.3. We first define the discrete analog of the parallel transport operator $\mathcal{U}(x ; C)$. We introduce an oriented contour $C$ on the lattice specified by a collection of links,

$$
C=\left\{\mu_{1}, \mu_{2}, \ldots, \mu_{n}\right\}
$$

where $\mu_{j}= \pm 1, \pm 2, \ldots, \pm D$. We define $U_{-\mu}(x)=U_{\mu}(x-\epsilon \hat{\mu})^{\dagger}$. The parallel transport operator can then be defined as

$$
\mathcal{U}(x ; C)=U_{\mu_{1}}(x) \star U_{\mu_{2}}\left(x+\epsilon \hat{\mu}_{1}\right) \star \cdots \star U_{\mu_{n}}\left(x+\epsilon \sum_{j=1}^{n-1} \hat{\mu}_{j}\right)
$$

As in the continuum, in order to construct star-gauge invariant observables out of the operator $\mathcal{U}(x ; C)$, we need a star-unitary function $S_{v}(x)$ with the property (2.25) for arbitrary functions $g(x)$ on the periodic lattice, where

$$
v=\epsilon \sum_{j=1}^{n} \hat{\mu}_{j} .
$$

One finds that a necessary and sufficient condition is given again by (2.46) with the solution $S_{v}(x)=\mathrm{e}^{i k_{\mu} x_{\mu}} \mathbb{1}_{p}$, where the loop momentum $k_{\mu}$ satisfies (2.47). In the present case, both $k$ and $v$ are quantized and periodic. Modding out by the periodicity, there are only the same, finite number of values that $k$ and $v$ can take. Therefore, it makes sense to ask whether or not (2.47) gives a one-to-one correspondence between $k$ and $v$. The answer is affirmative if and only if there exist $D \times D$ integer-valued matrices $J$ and $K$ which satisfy

$$
\frac{1}{\epsilon} \Sigma J-2 M K=\mathbb{1}_{D}
$$

where $M$ is the integral matrix introduced in (4.11). If this condition is not met, then there exist loop separation vectors $v$ for which there is no momentum $k$ satisfying (2.47), and for the other $v$ there is more than one value of $k$ satisfying (2.47). For example, let us consider 
the case with period matrix $\frac{1}{\epsilon} \Sigma=L \mathbb{1}_{D}$. If $L$ is an even integer, then (4.38) cannot be satisfied. If $L$ is odd and $M^{-1}$ is an integral matrix, then one can satisfy (4.38) by taking $J=\mathbb{1}_{D}$ and $K=\frac{L-1}{2} M^{-1}$.

Thus, for periodic gauge fields, one can construct a lattice regularization of noncommutative Yang-Mills theory with arbitrary gauge group rank $p$, period $\Sigma$, and noncommutativity parameter $\theta$. Due to Morita equivalence in the continuum, this means that we have a nonperturbative formulation of the general class of (twisted) continuum gauge theories that were described in Section 3.1. In this regard, it is important that Morita equivalence also holds at the level of observables as we discussed in Section 3.3. The problem with defining a discrete noncommutative $\mathrm{U}(p)$ gauge theory with multi-valued gauge fields directly is that one immediately encounters an obstacle to constructing a background abelian gauge field (3.6) on the lattice. As we discussed above, the relation (4.4) imposes a quantization constraint on the allowed momenta in the lattice discretization of noncommutative geometry. This would imply that the background tensor field $F$ be quantized proportionally to $\theta^{-1}$, and so the configuration (3.6) cannot be constructed on the lattice in general. However, as we have discussed, we circumvent this difficulty by using the noncommutative lattice gauge theory with single-valued gauge fields and Morita equivalence in the continuum to obtain a non-perturbative definition of all noncommutative Yang-Mills theories associated with constant curvature vector bundles over tori. Other problems with discretizing noncommutative geometry, such as the construction of an appropriate lattice Dirac operator, are discussed in Ref. 42].

\section{$5 \quad$ Explicit realizations of discrete noncommutative gauge theory}

The construction of the previous Section has been quite general, and we will now describe some concrete examples of discrete noncommutative Yang-Mills theory. Within the lattice formalism, we will demonstrate the Morita equivalence between commutative $\mathrm{U}(p)$ lattice gauge theory with fields obeying twisted boundary conditions and a noncommutative $\mathrm{U}\left(\tilde{p}_{0}\right)$ lattice gauge theory with fields obeying periodic boundary conditions. Using this property, we will further show that noncommutative gauge theory can be regularized by means of commutative lattice gauge theory with 't Hooft flux. As a special case, this construction includes a previous proposal for a concrete definition of noncommutative Yang-Mills theory using large $N$ reduced models [11]. This will further lead to the explicit finite dimensional matrix representations of the noncommutative torus that were discussed in the previous Section. 


\subsection{Discrete Morita equivalence}

In Section 4.2, we have shown that noncommutative Yang-Mills theories can be nonperturbatively regularized. The regularized theory is described by star-unitary gauge fields (4.28) on a lattice and the action (4.31) is defined in terms of lattice star-products. Although the theory is explicitly given in terms of a finite number of degrees of freedom, as it stands it is not very suitable for practical purposes, say for numerical studies such as Monte Carlo simulations. In this Subsection, we show further that noncommutative Yang-Mills theory can be nonperturbatively regularized by means of commutative lattice gauge theories with multi-valued gauge fields 32.

For this, we consider the lattice analog of Morita equivalence which we derived in Section 3.2. As we discussed in Section 4.2, there is a technical obstruction to constructing noncommutative Yang-Mills theory directly on the lattice for gauge fields of non-vanishing topological charge. We recall that the only obstacle was that the constant abelian background gauge field (3.6) does not generally have a momentum compatible with the restriction (4.4). This obstacle disappears, of course, for the commutative case $\theta_{\mu \nu}=0$. What we will prove in the lattice formulation is the Morita equivalence between commutative Yang-Mills theory with gauge fields obeying twisted boundary conditions and noncommutative Yang-Mills theory with periodic gauge fields, in arbitrary even dimension $D=2 d$. In fact, we will find that, for a given noncommutative Yang-Mills theory with arbitrary period matrix $\Sigma$ and deformation parameter $\Theta$ in the continuum, we can construct a family of commutative $\mathrm{U}(p)$ lattice gauge theories with 't Hooft flux whose sequence of Morita equivalent noncommutative theories converges to the target noncommutative field theory in the continuum limit. When $\Theta$ is irrational, the rank $p$ of the gauge group of the commutative theory must be sent to infinity as one takes the continuum limit. Combining this with the continuum Morita equivalence which connects two noncommutative Yang-Mills theories with periodic and twisted boundary conditions on the gauge fields, we will find that continuum noncommutative Yang-Mills theories, with either rational or irrational noncommutativity parameters and with or without twists in the boundary conditions on the gauge fields, can be regularized by means of commutative lattice gauge theory with 't Hooft flux.

We start with a commutative $\mathrm{U}(p)$ lattice gauge theory with fields obeying twisted boundary conditions. The action is

$$
S=-\frac{1}{g^{2}} \sum_{x} \sum_{\mu \neq \nu} \operatorname{tr}_{(p)}\left[U_{\mu}(x) U_{\nu}(x+\epsilon \hat{\mu}) U_{\mu}(x+\epsilon \hat{\nu})^{\dagger} U_{\nu}(x)^{\dagger}\right]
$$

where $U_{\mu}(x)$ are $\mathrm{U}(p)$ gauge fields satisfying the twisted boundary conditions

$$
U_{\mu}\left(x+\Sigma_{\nu a} \hat{\nu}\right)=\Omega_{a}(x) U_{\mu}(x) \Omega_{a}(x+\epsilon \hat{\mu})^{\dagger}
$$


with period matrix $\Sigma$. The transition functions $\Omega_{a}(x)$ are $\mathrm{U}(p)$ matrices which, for consistency of the constraints (5.2), must satisfy the cocycle condition

$$
\Omega_{a}\left(x+\Sigma_{\mu b} \hat{\mu}\right) \Omega_{b}(x)=\mathcal{Z}_{a b} \Omega_{b}\left(x+\Sigma_{\mu a} \hat{\mu}\right) \Omega_{a}(x),
$$

where $\mathcal{Z}_{a b}=\mathrm{e}^{2 \pi i \gamma_{a b} / p} \in \mathbb{Z}_{p}$. The antisymmetric matrix $\gamma$ has elements $\gamma_{a b} \in \mathbb{Z}$ representing the 't Hooft fluxes. As an explicit form of the transition functions, let us take the gauge choice

$$
\Omega_{a}(x)=1 \otimes \Gamma_{a}
$$

where $\Gamma_{a}$ are the $\mathrm{SU}(p)$ twist eaters obeying the Weyl-'t Hooft commutation relations (3.4). In order to satisfy (5.3) we must have $Q=\gamma$. Note that the gauge choice (5.4) corresponds to setting $\alpha=0$ in the continuum expression (3.2). The reason for this is that, as we discussed in Section 3.1, keeping both $\alpha$ and $\gamma$ non-zero in the commutative case is redundant. In other words, the abelian magnetic flux of a $\mathrm{U}(p)$ gauge field, which plays a very important role in Morita equivalences of noncommutative gauge theories [33], arises in the commutative case only via the corresponding 't Hooft flux 43. A similar consideration with non-vanishing $\alpha$ would lead us to the same final results. Due to the constraint (5.2), the gauge theory can be expressed in terms of lattice gauge fields $U_{\mu}(x)$ with $x_{\mu}$ lying in a unit cell of period $\Sigma$.

We will now show that the lattice field theory (5.1) with the constraint (5.2) is equivalent to a noncommutative $\mathrm{U}\left(\tilde{p}_{0}\right)$ lattice gauge theory with fields obeying periodic boundary conditions and the reduced rank $\tilde{p}_{0}$ defined by $(\overline{3.29})$. For this, we rewrite the lattice field theory (5.1) in terms of (finite dimensional) commutative operators. We write

$$
\begin{aligned}
& \hat{U}_{\mu}=\sum_{x} \hat{\Delta}(x) \otimes U_{\mu}(x) \\
& \hat{\Omega}_{a}=\sum_{x} \hat{\Delta}(x) \otimes \Omega_{a}(x),
\end{aligned}
$$

where the map $\hat{\Delta}(x)$ is defined by (4.18) and the operator $\hat{\Omega}_{a}$ is given explicitly by

$$
\hat{\Omega}_{a}=\mathbb{1} \otimes \Gamma_{a}
$$

The action (5.1) can then be written as

$$
S=-\frac{1}{g^{2}} \sum_{\mu \neq \nu} \operatorname{Tr} \operatorname{tr}{ }_{(p)}\left[\hat{U}_{\mu}\left(\hat{D}_{\mu} \hat{U}_{\nu} \hat{D}_{\mu}^{\dagger}\right)\left(\hat{D}_{\nu} \hat{U}_{\mu}^{\dagger} \hat{D}_{\nu}^{\dagger}\right) \hat{U}_{\nu}^{\dagger}\right]
$$

where $\hat{D}_{\mu}$ are the lattice shift operators (4.7), and the constraint (5.2) becomes

$$
\left(\hat{D}_{\nu}\right)^{\frac{1}{\epsilon} \Sigma_{\nu a}} \hat{U}_{\mu}\left(\hat{D}_{\nu}^{\dagger}\right)^{\frac{1}{\epsilon} \Sigma_{\nu a}}=\hat{\Omega}_{a} \hat{U}_{\mu} \hat{\Omega}_{a}^{\dagger}
$$


Proceeding exactly as in Section 3.1, one finds that the general solution to the constraint (5.9) is given by

$$
\hat{U}_{\mu}=\sum_{\vec{m}}\left(\prod_{a=1}^{D}\left(\hat{Z}_{a}^{\prime}\right)^{m_{a}}\right) \mathrm{e}^{-\pi i \sum_{a<b} \Theta_{a b}^{\prime} m_{a} m_{b}} \otimes u_{\mu}(\vec{m})
$$

where $u_{\mu}(\vec{m})$ is a $\tilde{p}_{0} \times \tilde{p}_{0}$ matrix and

$$
\hat{Z}_{a}^{\prime}=\mathrm{e}^{2 \pi i\left(\Sigma^{\prime}-1\right)_{a \mu} \hat{x}_{\mu}} \otimes \prod_{b=1}^{D}\left(\Gamma_{b}\right)^{B_{a b}}
$$

The $D \times D$ matrices $\Sigma^{\prime}$ and $\Theta^{\prime}$ are given by

$$
\begin{aligned}
& \Sigma^{\prime}=\Sigma \tilde{P} \\
& \Theta^{\prime}=-\tilde{P}^{-1} B^{\top},
\end{aligned}
$$

where the integral matrices $B$ and $\tilde{P}$ are defined in Section 3.2. Because of their dependence on the twist eating solutions, the operators (5.11) obey the commutation relations

$$
\begin{aligned}
\hat{Z}_{a}^{\prime} \hat{Z}_{b}^{\prime} & =\mathrm{e}^{-2 \pi i \Theta_{a b}^{\prime}} \hat{Z}_{b}^{\prime} \hat{Z}_{a}^{\prime} \\
\hat{D}_{\mu} \hat{Z}_{a}^{\prime} \hat{D}_{\mu}^{\dagger} & =\mathrm{e}^{2 \pi i\left(\Sigma^{\prime}-1\right)_{a \mu}} \hat{Z}_{a}^{\prime} .
\end{aligned}
$$

The commuation relations (5.14) are the same as (4.13) and (4.14) with the replacements $\hat{Z} \rightarrow \hat{Z}^{\prime}, \Theta \rightarrow \Theta^{\prime}$ and $\Sigma \rightarrow \Sigma^{\prime}$. The two matrices $\Sigma^{\prime}$ and $\Theta^{\prime}$ must satisfy the general constraint (4.16), which implies that $M=-\frac{1}{2 \epsilon} \Sigma B^{\top}$ must be an integer-valued matrix. The sum over $\vec{m}$ in $(5.10)$ can then be taken over $\mathbb{Z}^{D}$ modulo the periodicity $m_{a} \sim m_{a}+\frac{1}{\epsilon} \Sigma_{\mu a}^{\prime}$ with $\mu=1, \ldots, D$.

We now introduce a corresponding map $\hat{\Delta}^{\prime}\left(x^{\prime}\right)$ analogously to (4.18) and decompose the operator $\hat{U}_{\mu}$ in this new basis as

$$
\hat{U}_{\mu}=\sum_{x^{\prime}} \hat{\Delta}^{\prime}\left(x^{\prime}\right) \otimes U_{\mu}^{\prime}\left(x^{\prime}\right)
$$

Substituting (5.15) into (5.8), we arrive at the action

$$
S=-\frac{1}{g^{\prime 2}} \sum_{x^{\prime}} \sum_{\mu \neq \nu} \operatorname{tr}{ }_{\left(\tilde{p}_{0}\right)}\left[U_{\mu}^{\prime}\left(x^{\prime}\right) \star^{\prime} U_{\nu}^{\prime}\left(x^{\prime}+\epsilon \hat{\mu}\right) \star^{\prime} U_{\mu}^{\prime}\left(x^{\prime}+\epsilon \hat{\nu}\right)^{\dagger} \star^{\prime} U_{\nu}^{\prime}\left(x^{\prime}\right)^{\dagger}\right]
$$

where

$$
g^{\prime 2}=\frac{p}{\tilde{p}_{0}} g^{2}
$$

This shows that an ordinary $\mathrm{U}(p)$ lattice gauge theory with multi-valued gauge fields is equivalent to noncommutative $\mathrm{U}\left(\tilde{p}_{0}\right)$ lattice gauge theory with single-valued gauge fields and deformation parameter matrix (5.13). 
We now show that for any noncommutative Yang-Mills theory with given $\Theta^{\prime}$ and $\Sigma^{\prime}$ in the continuum and with gauge group $\mathrm{U}\left(\tilde{p}_{0}\right)$, we can construct a family of commutative $\mathrm{U}(p)$ lattice gauge theories with multi-valued gauge fields whose sequence of Morita equivalent theories converges to the target noncommutative gauge theory in the continuum limit. Let us first consider the case where the noncommutativity parameter matrix $\Theta^{\prime}$ given in the continuum is rational-valued. By using the $\operatorname{SL}(D, \mathbb{Z})$ transformation $\Theta^{\prime} \mapsto \Lambda \Theta^{\prime} \Lambda^{\top}$, where $\Lambda \in \mathrm{SL}(D, \mathbb{Z})$, we can rotate $\Theta^{\prime}$ into the canonical skew-diagonal form

$$
\Theta^{\prime}=\left(\begin{array}{ccccc}
0 & -\vartheta_{1}^{\prime} & & & \\
\vartheta_{1}^{\prime} & 0 & & & \\
& & \ddots & & \\
& & & 0 & -\vartheta_{d}^{\prime} \\
& & & \vartheta_{d}^{\prime} & 0
\end{array}\right)
$$

where the skew-eigenvalues $\vartheta_{i}^{\prime}$ are also rational numbers. Let us denote them by $\vartheta_{i}^{\prime}=-\frac{b_{i}}{\tilde{p}_{i}}$, where $b_{i}$ and $\tilde{p}_{i}$ are relatively prime integers for each $i=1, \ldots, d$. Then there exist integers $a_{i}$ and $\tilde{q}_{i}$ which satisfy (3.38). We can define the lattice rank $p$ by $(3.29)$ and the twist matrix $Q$ by (3.36). Since the matrix $\tilde{P}$ is invertible, we can approximate the period $\Sigma^{\prime}$ to arbitrary precision by the matrix $\Sigma \tilde{P}$ in the continuum limit $\epsilon \rightarrow 0$, while keeping fixed the lattice period $\frac{1}{\epsilon} \Sigma$ to an integer-valued matrix.

When $\Theta^{\prime}$ is irrational, one has to consider a rational-valued deformation parameter matrix $\Theta_{\epsilon}^{\prime}$ for each lattice spacing $\epsilon$, which converges to the given irrational numbers $\Theta_{\epsilon}^{\prime} \rightarrow \Theta^{\prime}$ as $\epsilon \rightarrow 0$. This means that the corresponding integers $b_{\epsilon, i}$ and $\tilde{p}_{\epsilon, i}$ should go to infinity in the continuum limit. Thus, the corresponding $p_{\epsilon}$ 's given by (3.29) should also go to infinity. We conclude that, in order to define a continuum noncommutative gauge theory with irrational noncommutativity parameters, the rank $p$ of the gauge group of the approximating commutative lattice gauge theory should be sent to infinity as one takes the continuum limit. Note that if one takes the 't Hooft (planar) limit by fixing $\lambda=g^{2} p$ as $p \rightarrow \infty$, then the coupling constant $g^{\prime}$ of the Morita equivalent noncommutative $\mathrm{U}\left(\tilde{p}_{0}\right)$ gauge theory is fixed according to (5.17). Therefore, in the 't Hooft limit, the lattice spacing $\epsilon$ should be taken to zero only after one takes the limit $p \rightarrow \infty$ and the limit should be accompanied by an appropriate renormalization of the 't Hooft coupling constant $\lambda$. However, in this limit the dimensionful noncommutativity parameters $\theta^{\prime}$ inevitably diverge. On the other hand, in order to have finite $\theta^{\prime}$ one should take a "double scaling limit" by sending $\epsilon \rightarrow 0$ together with the $p \rightarrow \infty$ limit in a correlated way. In this limit, non-planar Feynman diagrams survive in addition to the planar diagrams. Thus, although we have to take the large $p$ limit in order to obtain irrational $\Theta^{\prime}$, this limit should not be confused with the 't Hooft limit in general. 


\subsection{Twisted Eguchi-Kawai model}

We will now consider the construction of the previous Subsection in the special case where the period matrix is $\Sigma=\epsilon \mathbb{1}_{D}$. A one-site $\mathrm{U}(p)$ lattice gauge theory is just the Eguchi-Kawai model [26], and the fact that the boundary conditions are twisted as in (5.2) means that it is actually the twisted Eguchi-Kawai model [27]. To see this explicitly, we reduce the action (5.1) to a single point $x=0$ by using the constraints (5.2) to get

$$
U_{\mu}\left(\epsilon \delta_{\nu a} \hat{\nu}\right)=\Gamma_{a} U_{\mu}(0)\left(\Gamma_{a}\right)^{\dagger} .
$$

Substituting (5.19) into (5.1) at $x=0$ and using the commutation relations (3.4), we arrive at the action

$$
S=-\frac{1}{g^{2}} \sum_{\mu \neq \nu} \mathcal{Z}_{\mu \nu} \operatorname{tr}_{(p)}\left(V_{\mu} V_{\nu} V_{\mu}^{\dagger} V_{\nu}^{\dagger}\right)
$$

where $V_{\mu}=U_{\mu}(0) \Gamma_{\mu}, \mu=1, \ldots, D$, are $p \times p$ unitary matrices. This is the action of the twisted Eguchi-Kawai model, where the phase factor $\mathcal{Z}_{\mu \nu}=\mathrm{e}^{2 \pi i \gamma_{\mu \nu} / p}$ is called the "twist". Thus, the recent proposal [11] that the twisted large $N$ reduced model serves as a concrete definition of noncommutative Yang-Mills theory can be interpreted as the simplest example of Morita equivalence. The possibility of such an interpretation has also been suggested in Ref. [44].

Conversely, one can derive the explicit map from matrices in the twisted Eguchi-Kawai model to $\mathrm{U}\left(\tilde{p}_{0}\right)$ gauge fields on the noncommutative torus using the present formalism. For this, we decompose the matrices $V_{\mu}$ of the action (5.20) using the $\mathrm{SU}(p)$ twist eaters $\Gamma_{\mu}$ as

$$
V_{\mu}=U_{\mu} \Gamma_{\mu} .
$$

We then use the twisted boundary conditions (5.2) to generate from the $p \times p$ unitary matrix $U_{\mu}(0)=U_{\mu}$ a commutative $\mathrm{U}(p)$ lattice gauge field $U_{\mu}(x)$, and introduce the operator $\hat{U}_{\mu}$ by

(5.5). Then, using the expansion (5.15) and remembering that the original field theory is reduced to a point $x=0$, the $\mathrm{U}\left(\tilde{p}_{0}\right)$ gauge field $U_{\mu}^{\prime}\left(x^{\prime}\right)$ on the noncommutative torus can be given as

$$
\begin{aligned}
U_{\mu}^{\prime}\left(x^{\prime}\right)= & \operatorname{Tr}^{\prime}\left(\hat{\Delta}^{\prime}\left(x^{\prime}\right) \hat{U}_{\mu}\right) \\
= & \left.\frac{p}{\tilde{p}_{0}} \sum_{\vec{m}} \mathrm{e}^{\pi i \sum_{\mu<\nu} \Theta_{\mu \nu}^{\prime} m_{\mu} m_{\nu}} \mathrm{e}^{-2 \pi i\left(\Sigma^{\prime}-1\right.}\right)_{\mu \nu} m_{\mu} x_{\nu}^{\prime} \\
& \times \operatorname{tr}_{\left(\tilde{p}_{1} \otimes \cdots \otimes \tilde{p}_{d}\right)}\left[V_{\mu}\left(\Gamma_{\mu}\right)^{\dagger} \prod_{\nu=1}^{D}\left(\prod_{\rho=1}^{D}\left(\Gamma_{\rho}\right)^{B_{\nu \rho}}\right)^{m_{\nu}}\right] .
\end{aligned}
$$

Eq. (5.22) gives the mapping between the $p \times p$ unitary matrices $V_{\mu}$ of the twisted EguchiKawai model and noncommutative $\mathrm{U}\left(\tilde{p}_{0}\right)$ lattice gauge fields $U_{\mu}^{\prime}\left(x^{\prime}\right)$ with noncommutativity 
parameter matrix (5.13) and period matrix (5.12). This shows explicitly how, via Morita equivalence, large $N$ reduced gauge theories serve as a nonperturbative formulation of noncommutative Yang-Mills theory.

As in the case of general periods $\Sigma$ which we discussed in the previous Subsection, one can reproduce arbitrary dimensionless noncommutativity parameters $\Theta^{\prime}$ in the continuum limit. However, since $\Sigma=\epsilon \mathbb{1}_{D}$ in the present case, the induced period matrix $\Sigma^{\prime}$ in (5.12) is not the most general one. For example, in $D=2$ dimensions, one can obtain only a square torus, i.e. $\Sigma^{\prime}=\ell \mathbb{1}_{2}$, as the continuum spacetime. The rank $p$ of the gauge group of the reduced model must be sent to infinity even for rational $\Theta$, unlike the case of general $\Sigma$. We note also that the twisted Eguchi-Kawai model [26, 27] was originally proposed as a model which reproduces large $N$ gauge theory in the 't Hooft limit (or the planar limit). In the present interpretation, however, we have to take a different large $p$ limit, as we discussed in the previous subsection, whereby there are non-planar contributions. Therefore, although the matrix model is the same, the limiting procedure that one should take is different in the two interpretations. Indeed, the scaling of Wilson loops in such a non-planar large $p$ limit has been observed numerically for the two-dimensional Eguchi-Kawai model in Ref. 445].

Let us also comment on the compatibility of finite noncommutativity parameters and finite spacetime volume in the continuum limit. In Ref. [11], a particular choice of twist which corresponds to $q_{i}=L^{d-1}$ in eq. (3.27) with $p=L^{d}$ was taken, where $D=2 d$ is the dimension of spacetime. In that case, the integers $\tilde{p}_{i}$ and $\tilde{q}_{i}$ defined in (3.28) are given by $\tilde{p}_{i}=L$ and $\tilde{q}_{i}=1$. One can then take $a_{i}=0$ and $b_{i}=1$ to satisfy (3.38). Therefore, the volume of the torus is $(\epsilon L)^{D}$ and the dimensionful noncommutativity parameters are given by $\theta_{\mu \nu}^{\prime} \propto L \epsilon^{2}$. As a result, in order to keep the noncommutativity parameters finite in the continuum limit, one has to send the volume of the discrete periodic lattice to infinity.f In Ref. 22, this restriction was removed by considering a restricted twisted Eguchi-Kawai model obtained via the introduction of a quotient condition on the matrices which is the discrete analog of that which appears in the Connes-Douglas-Schwarz formalism [8]. However, the present general construction suggests a much simpler way to avoid this constraint, namely by choosing the twist as described below eq. (5.18).

We close this Section by remarking that the above derivations are independent of any representation of the operators $\hat{Z}_{a}^{\prime}$ and $\hat{D}_{\mu}$. This refines the approach of 222 whereby a specific finite dimensional representation of these operators was used to interpret the twisted Eguchi-Kawai model as noncommutative Yang-Mills theory. All we have used in the above construction is the equivalence of commutative and noncommutative gauge theories on the

\footnotetext{
${ }^{4}$ Indeed, in the two-dimensional Eguchi-Kawai model studied in Ref. 45, the scaling of Wilson loops was observed in the limit of large $L$ while keeping the parameter $L \epsilon^{2}$ fixed.
} 
lattice at the field theoretical level, without ever having to specify the representation of the operators used. To this end, we note that the above construction could have been obtained directly from the action (4.29) by using the representation of the operators in terms of the $p \times p$ matrices

$$
\begin{aligned}
& \hat{Z}_{a}^{\prime}=\prod_{b=1}^{D}\left(\Gamma_{b}\right)^{B_{a b}}, \\
& \hat{D}_{\mu}=\Gamma_{\mu} .
\end{aligned}
$$

These matrices clearly satisfy the commutation relations (5.14), and thereby constitute a finite dimensional representation of the noncommutative torus.

\section{Coupling to fundamental matter fields}

In this last Section we will consider noncommutative gauge theory coupled to matter fields in the fundamental representation of the gauge group. One advantage of the lattice formulation is that it allows a hopping parameter (or large mass) expansion of this theory which will enable us to clarify various aspects of the Wilson loops in noncommutative gauge theories that were constructed in Section 2.3. A peculiar property of these Wilson loops, in contrast to their commutative counterparts, is that star-gauge invariance does not require that the loops be closed, but it does require that the separation between their two endpoints is proportional to the total momentum of the loop. In the following we will clarify the physical meaning of these observables. This analysis will also demonstrate explicitly how these observables reduce smoothly to ordinary closed Wilson loops in the commutative limit. For this, we will first show that the effective action for the gauge field induced by integrating over the matter fields can be written as a sum over all star-gauge invariant observables associated with closed loops. We will then construct star-gauge invariant local operators out of the matter fields and evaluate their correlation functions, thereby demonstrating that the star-gauge invariant observables associated with open loops appear very naturally in this way.

We will also show that Morita equivalence holds in noncommutative Yang-Mills theory when it is coupled to fundamental matter fields. This Morita equivalence clarifies the interpretation of the perturbative beta-function which was calculated in Ref. [37. It also allows us to nonperturbatively define noncommutative Yang-Mills theory with fundamental matter fields, as we did in the matter-free case in Section 5.1. As a special case, we obtain the twisted Eguchi-Kawai model with fundamental matter fields as constructed in Ref. [36]. The main idea of these constructions is to introduce a number of flavours $N_{f}$ which is equal to (or, more generally, a multiple of) the number of colours, i.e. the rank of the gauge group. This 
enables us to impose a boundary condition on the matter fields using a rotation in flavour space to mimick the boundary condition for adjoint representation fields. For simplicity, we will consider only complex scalar matter fields. Fermionic matter fields can be treated in an analogous way.

\subsection{Properties of star-gauge invariant observables}

In this Subsection we will consider a discrete noncommutative torus and periodic boundary conditions on all fields. We introduce the operator

$$
\hat{\phi}=\sum_{x} \hat{\Delta}(x) \otimes \phi(x)
$$

where $\phi(x)$ is a complex scalar field in the fundamental representation of the gauge group $\mathrm{U}(p)$, and as always the lattice operator $\hat{\Delta}(x)$ is defined in (4.18). The action for the matter field can be written as

$$
S_{\text {matter }}=-\kappa \sum_{\mu}\left\{\operatorname{Tr}\left(\hat{\phi}^{\dagger} \hat{U}_{\mu} \hat{D}_{\mu} \hat{\phi} \hat{D}_{\mu}^{\dagger}\right)+\text { c.c. }\right\}+\operatorname{Tr}\left(\hat{\phi}^{\dagger} \hat{\phi}\right)
$$

and it is invariant under the transformation

$$
\begin{aligned}
\hat{\phi} & \mapsto \hat{g} \hat{\phi} \\
\hat{\phi}^{\dagger} & \mapsto \hat{\phi}^{\dagger} \hat{g}^{\dagger} \\
\hat{U}_{\mu} & \mapsto \hat{g} \hat{U}_{\mu} \hat{D}_{\mu} \hat{g}^{\dagger} \hat{D}_{\mu}^{\dagger}
\end{aligned}
$$

In terms of lattice fields, the action can be written as

$$
S_{\text {matter }}=-\kappa\left\{\sum_{x, \mu} \phi(x)^{\dagger} \star U_{\mu}(x) \star \phi(x+\epsilon \hat{\mu})+\text { c.c. }\right\}+\sum_{x} \phi(x)^{\dagger} \phi(x),
$$

and it is invariant under the star-gauge transformation

$$
\begin{aligned}
\phi(x) & \mapsto g(x) \star \phi(x) \\
\phi(x)^{\dagger} & \mapsto \phi(x)^{\dagger} \star g(x)^{\dagger} \\
U_{\mu}(x) & \mapsto g(x) \star U_{\mu}(x) \star g(x+\epsilon \hat{\mu})^{\dagger} .
\end{aligned}
$$

When we integrate over the matter field $\phi(x)$, we make an expansion with respect to the hopping parameter $\kappa$. This will require the computation of various correlation functions of the scalar fields, which can be calculated by using the formulae

$$
\begin{gathered}
\sum_{x, y} \phi(x)^{\dagger} \star F(x) \star\left\langle\phi(x+v) \phi(y)^{\dagger}\right\rangle_{\kappa=0} \star G(y) \star \phi(y+u) \\
=\sum_{x} \phi(x)^{\dagger} \star F(x) \star G(x+v) \star \phi(x+v+u),
\end{gathered}
$$




$$
\sum_{x}\left\langle\phi(x)^{\dagger} \star F(x) \star \phi(x+v)^{\dagger}\right\rangle_{\kappa=0}=\delta_{v, 0} \sum_{x} F(x) .
$$

Throughout this section, the brackets $\langle\cdots\rangle$ denote the vacuum expectation value for fixed gauge background, i.e. we integrate over the matter fields only. The suffix $\kappa=0$ means that the hopping parameter is set to zero in the matter field action (6.4). Star-gauge invariance requires that the lattice fields $F(x)$ and $G(x)$ transform as

$$
\begin{aligned}
& F(x) \mapsto g(x) \star F(x) \star g(x+v)^{\dagger} \\
& G(y) \mapsto g(y) \star G(y) \star g(y+u)^{\dagger}
\end{aligned}
$$

under the star-gauge transformation (6.5).

Let us first consider the effective action $\Gamma_{\text {eff }}[U]$ for the gauge field $U_{\mu}(x)$ induced by the integration over $\phi(x)$. Using the hopping parameter expansion, it can be given as

$$
\Gamma_{\text {eff }}[U]=-\ln \left[\sum_{n=0}^{\infty} \frac{\kappa^{n}}{n !}\left\langle\left(\sum_{x, \mu} \phi(x)^{\dagger} \star U_{\mu}(x) \star \phi(x+\epsilon \hat{\mu})+\text { c.c. }\right)^{n}\right\rangle_{\kappa=0}\right] .
$$

Integrating over the matter field using Wick's theorem and the formulae (6.6) and (6.7), we obtain

$$
\Gamma_{\text {eff }}[U]=\sum_{C} \frac{\kappa^{L(C)}}{L(C)} \sum_{x} \operatorname{tr}_{(p)}(\mathcal{U}(x ; C))
$$

where $\sum_{C}$ is the sum over all closed loops (with and without self-intersection) on the lattice, and $L(C)$ denotes the number of links in the contour $C$. In this way, we encounter the star-gauge invariant observables associated with closed loops.

Let us now consider star-gauge invariant observables involving matter fields such as

$$
G_{1}[f]=\left\langle\sum_{x} \phi(x)^{\dagger} \star \phi(x) \star f(x)\right\rangle,
$$

which is star-gauge invariant for arbitrary functions $f(x)$ on the lattice. The lattice field $f(x)$ can be regarded as the wavefunction of the composite operator $\phi(x)^{\dagger} \star \phi(x)$. We first perform a Fourier transformation of $f(x)$ and express the observable 6.11) as

$$
G_{1}[f]=\sum_{\vec{k}} \tilde{f}(k)\left\langle\sum_{x} \phi(x)^{\dagger} \star \mathrm{e}^{i k_{\mu} x_{\mu}} \mathbb{1}_{p} \star \phi(x+v)\right\rangle,
$$

where

$$
v_{\mu}=\theta_{\mu \nu} k_{\nu} .
$$

Here we have used the fact that the plane wave $\mathrm{e}^{i k_{\mu} x_{\mu}} \mathbb{1}_{p}$ acts as a translation operator in the noncommutative field theory as explained in (2.25)-(2.27). Integrating over the matter 
field using the hopping parameter expansion, Wick's theorem, and the formulae (6.6) and (6.7), we arrive at

$$
G_{1}[f]=\sum_{\vec{k}} \tilde{f}(k) \sum_{C_{v}} \kappa^{L\left(C_{v}\right)} \sum_{x} \operatorname{tr}_{(p)}\left(\mathcal{U}\left(x ; C_{v}\right) \star \mathrm{e}^{i k_{\mu} x_{\mu}} \mathbb{1}_{p}\right),
$$

where $\sum_{C_{v}}$ denotes the sum over all loops on the lattice starting from the origin and ending at the lattice point (6.13). Thus we find the star-gauge invariant observables encountered in Section 2.3 associated with open loops. In the commutative case $\theta_{\mu \nu}=0$, the separation vector (6.13) of the loops vanishes independently of their momenta $k_{\mu}$. Then the sum over loops in (6.14) contains only closed loops. The sum over $\vec{k}$ can therefore be done explicitly reproducing the wavefunction $f(x)$. In the noncommutative case, however, the sum over loops depends on $k_{\mu}$ and we cannot reverse the order of the first two sums in (6.14). The separation vector $v_{\mu}$ of the two ends of the loop $C_{v}$ grows with its momentum $k_{\mu}$. This is a characteristic phenomenon in noncommutative field theories, and it is another signal of the UV/IR mixing. If one would like to have a higher resolution in one direction, say in the $\mu=1$ direction, by increasing the momentum $k_{1}$, then the object will extend in the other directions proportionally to $\theta_{\mu 1} k_{1}$. Thus the size of the object depends on its momentum. On the other hand, if one considers the case where $\tilde{f}(k)$ is supported on finite momenta $k_{\mu}$, then one can take the $\theta_{\mu \nu} \rightarrow 0$ limit smoothly, reproducing the commutative case.

Let us next consider a two-point function of the composite operators which is defined as

$$
G_{2}[f, g]=\left\langle\sum_{x} \phi(x)^{\dagger} \star \phi(x) \star f(x) \cdot \sum_{y} \phi(y)^{\dagger} \star \phi(y) \star g(y)\right\rangle .
$$

The connected part of this correlation function can be readily computed as before to give

$$
\begin{aligned}
G_{2}^{(\text {conn })}[f, g] \stackrel{\text { def }}{=} & G_{2}[f, g]-G_{1}[f] G_{1}[g] \\
= & \sum_{\vec{k}, \vec{p}} \tilde{f}(k) \tilde{g}(p) \sum_{C_{u}, C_{v}^{\prime}} \kappa^{L\left(C_{u}\right)+L\left(C_{v}^{\prime}\right)} \\
& \times \sum_{x, y} \operatorname{tr}_{(p)}\left(\mathcal{U}\left(x ; C_{u}\right) \star \mathrm{e}^{i p_{\nu} y_{\nu}} \mathbb{1}_{p} \star \mathcal{U}\left(y ; C_{v}^{\prime}\right) \star \mathrm{e}^{i k_{\mu} x_{\mu}} \mathbb{1}_{p}\right),
\end{aligned}
$$

where the double sum over contours in (6.16) is restricted in such a way that the parallel transport operator $\mathcal{U}\left(x ; C_{u}\right)$ goes from $x$ to $y+u$ and $\mathcal{U}\left(y ; C_{v}^{\prime}\right)$ from $y$ to $x+v$. The separation vectors $v_{\mu}$ and $u_{\mu}$ are related to the momenta $k_{\mu}$ and $p_{\mu}$ respectively by $u_{\mu}=\theta_{\mu \nu} p_{\nu}$ and $v_{\mu}=\theta_{\mu \nu} k_{\nu}$. As with the one-point function (6.14), the sums over $\vec{k}$ and $\vec{p}$ can be done explicitly in the commutative limit, reproducing the wavefunctions $f(x)$ and $g(y)$. In the noncommutative case, the sums over contours in (6.16) depend on the momenta $\vec{k}$ and $\vec{p}$ and one cannot interchange the momentum and loop sums. Note that the two parallel transport 
operators in (6.16) do not form a closed loop. The difference between the endpoint of one and the starting point of the other are given by $u_{\mu}$ and $v_{\mu}$. If one increases the momenta $k_{\mu}$ or $p_{\mu}$, then the sizes $u_{\mu}$ or $v_{\mu}$ also increase.

The considerations in this section illustrate that the star-gauge invariant observables constructed in Section 2.3 do indeed play the same fundamental role as ordinary Wilson loops do in commutative gauge theories. As the knowledge of all Wilson loop correlators would give all the information about the quantum gauge theory coupled to matter fields, so do the star-gauge invariant observables in the noncommutative case. In particular, this result implies that the issue of whether or not a noncommutative gauge theory is confining can be addressed by searching for the area law behaviour of a star-gauge invariant observable associated with a closed loop. We have also seen explicitly how these observables reduce smoothly to ordinary Wilson loops in the commutative limit for fixed gauge background.

\subsection{Morita equivalence with fundamental matter fields}

In this section, we will describe Morita equivalence of noncommutative Yang-Mills theories coupled to fundamental matter fields. We consider the setup of Section 5.1. We start with commutative $\mathrm{U}(p)$ gauge theory and introduce $N_{f}$ flavours of matter fields in the fundamental representation of the gauge group. We represent it as a matrix $\Phi(x)_{i J}$, where $i=1, \ldots, p$ is the colour index and $J=1, \ldots, N_{f}$ is the flavour index. The spacetime is discretized as $x_{\mu} \in \epsilon \mathbb{Z}$. For the present purposes, it is essential to take $N_{f}$ to be an integer multiple of $p$. In what follows, we will assume that $N_{f}=p$ for simplicity. Then, $\Phi(x)_{i J}$ becomes a $p \times p$ complex-valued matrix, i.e. an element of $\operatorname{gl}(p, \mathbb{C})$.

The action for the gauge field is given by (5.1). The action for the matter field is

$$
S_{\text {matter }}=-\kappa\left\{\sum_{x, \mu} \operatorname{tr}_{(p)}\left(\Phi(x)^{\dagger} U_{\mu}(x) \Phi(x+\epsilon \hat{\mu})\right)+\text { c.c. }\right\}+\sum_{x} \operatorname{tr}_{(p)}\left(\Phi(x)^{\dagger} \Phi(x)\right) .
$$

This matter-coupled gauge theory is invariant under the gauge transformation

$$
\begin{aligned}
U_{\mu}(x) & \mapsto g(x) U_{\mu}(x) g(x+\epsilon \hat{\mu}) \\
\Phi(x) & \mapsto g(x) \Phi(x) \\
\Phi(x)^{\dagger} & \mapsto \Phi(x)^{\dagger} g(x)^{\dagger} .
\end{aligned}
$$

The action (6.17) also possesses the global $\mathrm{U}\left(N_{f}\right)$ flavour symmetry

$$
\Phi(x) \mapsto \Phi(x) g^{\prime} \quad ; \quad \Phi(x)^{\dagger} \mapsto g^{\prime \dagger} \Phi(x)^{\dagger},
$$

where $g^{\prime} \in \mathrm{U}\left(N_{f}\right)$. 
We impose twisted boundary conditions on the fields $\Phi(x)$ and $U_{\mu}(x)$ given by

$$
\begin{aligned}
U_{\mu}\left(x+\Sigma_{\nu a} \hat{\nu}\right) & =\Gamma_{a} U_{\mu}(x)\left(\Gamma_{a}\right)^{\dagger} \\
\Phi\left(x+\Sigma_{\nu a} \hat{\nu}\right) & =\Gamma_{a} \Phi(x)\left(\Gamma_{a}\right)^{\dagger}
\end{aligned}
$$

where $\Sigma$ is the period matrix and $\Gamma_{a}$ are the twist-eaters satisfying the commutation relations (3.4). Note that in (6.20), the $\Gamma_{a}$ acting on the left of $\Phi(x)$ represent a (global) gauge transformation, whereas the $\left(\Gamma_{a}\right)^{\dagger}$ acting on the right represent a rotation in flavour space. This is the trick to introducing the fundamental representation of the gauge group in Morita equivalence transformations. Even though the matter fields define fundamental sections of the given gauge bundle over the noncommutative torus (see (6.18)), we can exploit the global $\mathrm{SU}\left(N_{f}\right)$ flavour symmetry to mimick the boundary conditions for adjoint representation fields. Such an idea first appeared in Ref. [46] in the context of supersymmetric field theories.

Rewriting the fields in terms of operators and solving the constraints (6.20) as we did in Section 5.1, we find that the resulting field theory is noncommutative $\mathrm{U}\left(\tilde{p}_{0}\right)$ lattice gauge theory, on a lattice with period matrix $\Sigma^{\prime}=\Sigma \tilde{P}$ and with dimensionless noncommutativity

parameters given by $\Theta^{\prime}=-\tilde{P}^{-1} B^{\top}$, coupled to $\tilde{p}_{0}$ flavours of matter fields in the fundamental representation of $\mathrm{U}\left(\tilde{p}_{0}\right)$. When $N_{f}=n_{f} p$, we obtain $n_{f} \tilde{p}_{0}$ flavours in the Morita equivalent noncommutative field theory. The one-loop beta-function of this theory for $\tilde{p}_{0}=1$ has been calculated in Ref. [37]. The contribution of fundamental matter fields to the oneloop beta-function of noncommutative $\mathrm{U}\left(\tilde{p}_{0}\right)$ Yang-Mills theory with $n_{f}$ flavours coincides up to a finite rescaling of the Yang-Mills coupling constant with that in commutative $\mathrm{U}(p)$ Yang-Mills theory with $N_{f}=n_{f} p$ flavours, since one-loop Feynman diagrams involving fundamental matter fields are always planar. This coincidence can now be understood as a manifestation at the one-loop level of the Morita equivalence we have found in this Section.

As we did in Section 5.2, we can set $\Sigma=\epsilon \mathbb{1}_{D}$ to obtain the twisted Eguchi-Kawai model coupled to matter fields. This is the model which was introduced in Ref. 36 as a model which reproduces large $N$ gauge theory with $N_{f}$ flavours of matter in the Veneziano limit $N_{f} \sim N \rightarrow \infty$. Here we have found that the same model with $N_{f}=n_{f} N$ can be interepreted as noncommutative $\mathrm{U}\left(\tilde{p}_{0}\right)$ gauge theory with periodic fields including $n_{f} \tilde{p}_{0}$ flavours of matter fields in the fundamental representation of the gauge group $\mathrm{U}\left(\tilde{p}_{0}\right)$.

\section{Acknowledgements}

The work of J.A. and Y.M. is supported in part by MaPhySto founded by the Danish National Research Foundation. Y.M. is sponsored in part by the Danish National Bank. J.N. is supported by the Japan Society for the Promotion of Science as a Research Fellow 
Abroad. The work of R.J.S. is supported in part by the Danish Natural Science Research Council.

\section{References}

[1] A. Connes, Noncommutative Geometry (Academic Press, 1994);

G. Landi, An Introduction to Noncommutative Spaces and their Geometries (SpringerVerlag, 1997);

J.C. Várilly, physics/9709045;

J. Madore, An Introduction to Noncommutative Geometry and its Physical Applications (Cambridge University Press, 1999).

[2] J. Fröhlich, O. Grandjean and A. Recknagel, in: Quantum Symmetries, eds. A. Connes, K. Gawędzki and J. Zinn-Justin, Les Houches Session 64 (North-Holland, 1998), p. 221, hep-th/9706132.

[3] T. Yoneya, hep-th/0004074, and references therein.

[4] G. Veneziano, Europhys. Lett. 2 (1986) 199;

D.J. Gross and P.F. Mende, Nucl. Phys. B303 (1988) 407;

D. Amati, M. Ciafaloni and G. Veneziano, Phys. Lett. B216 (1989) 41.

[5] J. Fröhlich and K. Gawędzki, CRM Proc. Lecture Notes 7 (1994) 57, hep-th/9310187;

P.-M. Ho and Y.-S. Wu, Phys. Lett. B398 (1997) 52, hep-th/9611233;

A.H. Chamseddine, Phys. Lett. B400 (1997) 87, hep-th/9701096; Phys. Rev. D56 (1997) 3555, hep-th/9705153;

F. Lizzi and R.J. Szabo, Phys. Rev. Lett. 79 (1997) 3581, hep-th/9706107; Commun. Math. Phys. 197 (1998) 667, hep-th/9707202; J. High Energy Phys. Conf. Proc. corfu98/073, hep-th/9904064.

[6] T. Banks, W. Fischler, S.H. Shenker and L. Susskind, Phys. Rev. D55 (1997) 5112, hep-th/9610043.

[7] N. Ishibashi, H. Kawai, Y. Kitazawa and A. Tsuchiya, Nucl. Phys. B498 (1997) 467, hep-th/9612115.

[8] A. Connes, M.R. Douglas and A. Schwarz, J. High Energy Phys. 9802 (1998) 003, hep-th/9711162.

[9] M.R. Douglas and C.M. Hull, J. High Energy Phys. 9802 (1998) 008, hep-th/9711165;

Y.-K.E. Cheung and M. Krogh, Nucl. Phys. B528 (1998) 185, hep-th/9803031;

D. Bigatti, Phys. Lett. B451 (1999) 324, hep-th/9804120;

F. Ardalan, H. Arfaei and M.M. Sheikh-Jabbari, J. High Energy Phys. 9902 (1999) 016, hep-th/9810072;

M.M. Sheikh-Jabbari, Phys. Lett. B450 (1999) 129, hep-th/9810179;

C.-S. Chu and P.-M. Ho, Nucl. Phys. B550 (1999) 151, hep-th/9812219;

V. Schomerus, J. High Energy Phys. 9906 (1999) 030, hep-th/9903205;

D. Bigatti and L. Susskind, hep-th/9908056.

[10] N. Seiberg and E. Witten, J. High Energy Phys. 9909 (1999) 032, hep-th/9908142.

[11] H. Aoki, N. Ishibashi, S. Iso, H. Kawai, Y. Kitazawa and T. Tada, Nucl. Phys. B565 (2000) 176, hep-th/9908141.

[12] H.S. Snyder, Phys. Rev. 71 (1947) 38; 68. 
[13] T. Filk, Phys. Lett. B376 (1996) 53.

[14] J.C. Várilly and J.M. Gracia-Bondía, Int. J. Mod. Phys. A14 (1999) 1305, hep-th/9804001;

M. Chaichian, A. Demichev and P. Presnajder, Nucl. Phys. B567 (2000) 360, hep-th/9812180;

C.P. Martín and D. Sanchez-Ruiz, Phys. Rev. Lett. 83 (1999) 476, hep-th/9903077;

M.M. Sheikh-Jabbari, J. High Energy Phys. 9906 (1999) 015, hep-th/9903107;

T. Krajewski and R. Wulkenhaar, hep-th/9903187.

[15] N. Ishibashi, S. Iso, H. Kawai and Y. Kitazawa, hep-th/9910004, to appear in Nucl. Phys. B.

[16] S. Minwalla, M. Van Raamsdonk and N. Seiberg, J. High Energy Phys. 0002 (2000) 020, hep-th/9912072.

[17] I.Ya. Aref'eva, D.M. Belov and A.S. Koshelev, hep-th/0001215; hep-th/0003176;

A. Matusis, L. Susskind and N. Toumbas, hep-th/0002075;

M. Van Raamsdonk and N. Seiberg, J. High Energy Phys. 0003 (2000) 035, hep-th/0002186;

F. Zamora, hep-th/0004085.

[18] J. McGreevy, L. Susskind and N. Toumbas, hep-th/0003075;

O. Andreev and H. Dorn, hep-th/0003113:

Y. Kiem and S. Lee, hep-th/0003145;

A. Bilal, C.-S. Chu and R. Russo, hep-th/0003180;

J. Gomis, M. Kleban, T. Mehen, M. Rangamani and S.H. Shenker, hep-th/0003215;

A. Rajaraman and M. Rozali, hep-th/0003227;

H. Liu and J. Michelson, hep-th/0004013;

P.-M. Ho and M. Li, hep-th/0004072;

G. Arcioni, J.L.F. Barbon, J. Gomis and M.A. Vazquez-Mozo, hep-th/0004080.

[19] N. Ishibashi, S. Iso, H. Kawai and Y. Kitazawa, hep-th/0004038.

[20] R. Gopakumar, S. Minwalla and A. Strominger, hep-th/0003160.

[21] I. Bars and D. Minic, hep-th/9910091.

[22] J. Ambjørn, Y.M. Makeenko, J. Nishimura and R.J. Szabo, J. High Energy Phys. 9911 (1999) 029, hep-th/9911041.

[23] S. Iso, H. Kawai and Y. Kitazawa, hep-th/0001027.

[24] J. Ambjørn, Y.M. Makeenko, J. Nishimura and R.J. Szabo, hep-th/0002158, to appear in Phys. Lett. B.

[25] D.B. Fairlie and C.K. Zachos, Phys. Lett. B224 (1989) 101;

M. Li, Nucl. Phys. B499 (1997) 149, hep-th/9612222;

T. Curtright, D.B. Fairlie and C.K. Zachos, Phys. Lett. B405 (1997) 37, hep-th/9704037;

D.B. Fairlie, Mod. Phys. Lett. A13 (1998) 263, hep-th/9707190.

[26] T. Eguchi and H. Kawai, Phys. Rev. Lett. 48 (1982) 1063.

[27] A. Gonzalez-Arroyo and M. Okawa, Phys. Lett. B120 (1983) 174; Phys. Rev. D27 (1983) 2397;

T. Eguchi and R. Nakayama, Phys. Lett. B122 (1983) 59. 
[28] W. Taylor, Phys. Lett. B394 (1997) 283, hep-th/9611042;

O.J. Ganor, S. Ramgoolam and W. Taylor, Nucl. Phys. B492 (1997) 191, hep-th/9611202.

[29] K.G. Wilson, Phys. Rev. D10 (1974) 2445.

[30] H. Weyl, The Theory of Groups and Quantum Mechanics (Dover, 1950).

[31] A. Dimakis, F. Müller-Hoissen and T. Striker, J. Phys. A26 (1993) 1927;

A.P. Balachandran, G. Bimonte, E. Ercolessi, G. Landi, F. Lizzi, G. Sparano and P. Teotonio-Sobrinho, Nucl. Phys. B (Proc. Suppl.) 37C (1995) 20, hep-th/9403067; J. Geom. Phys. 18 (1996) 163, hep-th/9510217;

A.P. Balachandran, G. Bimonte, E. Ercolessi, G. Landi, F. Lizzi and P. TeotonioSobrinho, J. Geom. Phys. 24 (1998) 353, hep-lat/9604012.

[32] G. 't Hooft, Nucl. Phys. B153 (1979) 141; Commun. Math. Phys. 81 (1981) 267.

[33] M. Rieffel and A. Schwarz, Int. J. Math. 10 (1999) 189, math.QA/9803057;

P.-M. Ho, Phys. Lett. B434 (1998) 41, hep-th/9803166;

A. Schwarz, Nucl. Phys. B534 (1998) 720, hep-th/9805034;

G. Landi, F. Lizzi and R.J. Szabo, Commun. Math. Phys. 206 (1999) 603, hep-th/9806099;

D. Brace, B. Morariu and B. Zumino, Nucl. Phys. B545 (1999) 192, hep-th/9810099;

C. Hofman and E. Verlinde, Nucl. Phys. B547 (1999) 157, hep-th/9810219;

B. Pioline and A. Schwarz, J. High. Energy Phys. 9908 (1999) 021, hep-th/9908019;

E. Kim, H. Kim, N. Kim, B.-H. Lee, C.-Y. Lee and H.S. Yang, hep-th/9912272.

[34] K. Okuyama, J. High Energy Phys. 0003 (2000) 016, hep-th/9910138;

P. Watts, Phys. Lett. B474 (2000) 295, hep-th/9911026;

T. Lee, Phys. Lett. B478 (2000) 313, hep-th/9912038;

O. Andreev and H. Dorn, Phys. Lett. B476 (2000) 402, hep-th/9912070.

[35] A.Yu. Alekseev and A.G. Bytsko, hep-th/0002101.

[36] S.R. Das, Phys. Lett. B132 (1983) 155.

[37] M. Hayakawa, Phys. Lett. B478 (2000) 394, hep-th/9912094; hep-th/9912167.

[38] I. Chepelev and R. Roiban, hep-th/9911098;

I.Ya. Aref'eva, D.M. Belov and A.S. Koshelev, Phys. Lett. B476 (2000) 431, hep-th/9912075.

[39] P. van Baal and B. van Geemen, J. Math. Phys. 27 (1986) 455;

D.R. Lebedev and M.I. Polikarpov, Nucl. Phys. B269 (1986) 285.

[40] G. Landi, F. Lizzi and R.J. Szabo, hep-th/9912130.

[41] F. Lizzi and R.J. Szabo, Chaos Solitons Fractals 10 (1999) 445, hep-th/9712206.

[42] G. Bimonte, F. Lizzi and G. Sparano, Phys. Lett. B341 (1994) 139, hep-lat/9404007;

M. Göckeler and T. Schücker, Phys. Lett. B434 (1998) 80, hep-th/9805077.

[43] D.R. Lebedev, M.I. Polikarpov and A.A. Rosly, Nucl. Phys. B325 (1989) 138.

[44] A. Hashimoto and N. Itzhaki, J. High Energy Phys. 9912 (1999) 007, hep-th/9911057.

[45] T. Nakajima and J. Nishimura, Nucl. Phys. B528 (1998) 355, hep-th/9802082.

[46] E. Cohen and C. Gomez, Nucl. Phys. B223 (1983) 183. 